\title{
Heavy baryon spectrum on the lattice with NRQCD bottom and HISQ lighter quarks
}

\author{
Protick Mohanta and Subhasish Basak® \\ School of Physical Sciences, National Institute of Science Education and Research, \\ Homi Bhabha National Institute, Odisha 752050, India
}

(Received 9 November 2019; accepted 21 April 2020; published 12 May 2020)

\begin{abstract}
We determine the mass spectra of heavy baryons containing one or more bottom quarks along with their hyperfine splittings and various mass differences on MILC $2+1$ Asqtad lattices at three different lattice spacings. NRQCD action is used for bottom quarks whereas relativistic HISQ action for the lighter up/ down, strange, and charm quarks. We consider all possible combinations of bottom and lighter quarks to construct the bottom baryon operators for the states $J^{P}=1 / 2^{+}$and $3 / 2^{+}$.
\end{abstract}

DOI: 10.1103/PhysRevD.101.094503

\section{INTRODUCTION}

Lattice QCD has been extensively employed to study $B$ physics phenomenology, especially the decay constants and mixing parameters needed for CKM matrix elements and the mass differences in the meson sector [1]. The $B$ mesons spectroscopy and mass splittings have undergone thorough investigations on lattice, see [1] and references therein, with an increasing impact on heavy flavor phenomenology; see, for instance, [2]. However, studying heavy baryons with bottom quark(s) on lattice is relatively a recent pursuit. Some of the early studies of heavy baryons on lattice can be found in [3-7]. Of late, a slew of low lying $J^{P}=1 / 2^{+}$ bottom baryons, such as $\Lambda_{b}, \Sigma_{b}, \Xi_{b}^{\prime}$, and $\Omega_{b}$, have made entries in Particle Data Group (PDG) [8]. The possibilities of the discoveries of $J^{P}=3 / 2^{+}$are rather high, whereas doubly and triply bottom baryons are right now beyond the reaches of present experiments. In this state, lattice QCD can provide an insight into the masses, mass splittings, and other properties of such bottom baryons from the first principle. To this end, quite a few lattice investigations of heavy baryons containing one, two, or three bottom quarks have been undertaken using a range of light quark actions [9-11]. For an extensive list on contemporary lattice literature on heavy baryons, see [11].

These studies on heavy hadrons with bottom quark(s) are largely made possible by the use of nonrelativistic QCD action, proposed and formulated in $[12,13]$, because of the well-known fact that the current lattice spacings, even for as low as $0.045 \mathrm{fm}$, render $a m_{b} \gtrsim 1$. Although almost all of

Published by the American Physical Society under the terms of the Creative Commons Attribution 4.0 International license. Further distribution of this work must maintain attribution to the author(s) and the published article's title, journal citation, and DOI. Funded by SCOAP. the above studies employed different heavy quark actions for charm quark, HISQ action [14] is becoming an increasingly popular choice for the charm quark. This approach of simulating bottom quark with NRQCD and the rest of the quarks i.e., charm, strange, and up/down with HISQ for calculation of bottom baryon spectra has been adopted in this work.

In this paper, we present our lattice QCD results of heavy baryons involving one and two bottom quarks. We consider all possible combinations of bottom quark(s) with charm, strange, and up/down lighter quarks of the form $(l b b),(l l b)$, and $\left(l_{1} l_{2} b\right)$, where $b$ is the bottom quark and $l$ are the lighter charm, strange, and up/down quarks. We are addressing the charm quark as "light" quark in the sense that we have used relativistic action for it. The action for the lighter quarks is HISQ [14] and NRQCD [12,13] for the bottom. We discuss these actions in Sec. II. The propagators generated using nonrelativistic and relativistic actions are required to be combined to construct baryon states of appropriate quantum numbers. A discussion to achieve this combination is spread over both Secs. II and III. The bottom baryon operators are described in details in Sec. III. In the following Sec. IV, we present the simulation details including the lattice ensembles used, various parameters, and tuning of different quark masses. The lattice calculations are carried out at three different lattice spacings with fixed $m_{u / d} / m_{s}$ value and several quark masses. We assemble our bottom baryon spectrum results along with hyperfine and various other mass splittings in the Sec. V. Finally, we conclude and summarize in Sec. VI, which also includes a comparison of our results to the existing ones.

\section{QUARK ACTIONS}

As of now, the bottom (b) quark masses are not small, i.e., $a m_{b} \nless 1$ in units of the lattice spacings available. 
The use of improved NRQCD is the action of choice for the $b$ quarks. We have used $\mathcal{O}\left(v^{6}\right)$ NRQCD action in this paper. The charm (c) quark is also similarly heavy enough for existing lattices, but the Fermilab proposal [15] made it possible to work with relativistic actions for the $c$ quark, provided we trade the pole mass with the kinetic mass. Subsequently, HISQ action [14] became available for relativistic $c$ quark. In this paper, we choose HISQ action for the $c$ quark along with $s$ and $u / d$ quarks. In this work, as because we use the same relativistic HISQ action for all quarks except the bottom, we use the word light quarks to refer to $c, s$, and $u / d$ quarks. This is similar to what has been done in [16] for the $B$ meson states calculation. Besides, one of the big advantages of this choice of action is the ability to use the MILC code [17] for this bit.

\section{A. NRQCD action and $b$ quark}

In order to perform a lattice QCD computation of hadrons containing bottom quarks in publicly available relatively coarse lattices, NRQCD $[12,13]$ is perhaps the most suitable and widely used quark action for the bottom. As is understood, the typical velocity of a $b$ quark inside a hadron is nonrelativistic. Comparison of masses of bottomonium states to the mass of $b$ quark supports the fact that the velocity of $b$ quark inside hadron $\left(v^{2} \sim 0.1\right)$ is much smaller than the bottom mass. For example $M_{\Upsilon}=$ $9460 \mathrm{MeV}$ whereas $2 \times m_{b}=8360 \mathrm{MeV}$ in the $\overline{\mathrm{MS}}$ scheme. For bottom hadrons containing lighter valence quarks, the velocity of the bottom quark is even smaller. This allows us to study the $b$ quark with a nonrelativistic effective field theory. NRQCD will remain the action of choice for the $b$ quark until finer lattices with $a m_{b}<1$ become widely available.

In NRQCD, the upper and lower components of the Dirac spinor decouple, and the $b$ quark is described by a two component spinor field, denoted by $\psi_{h}$. The NRQCD Lagrangian has the following form:

$\mathcal{L}=\psi_{h}^{\dagger}(\vec{x}, t)\left[U_{4}(x) \psi_{h}(\vec{x}, t+1)-\psi_{h}(\vec{x}, t)+a H \psi_{h}(\vec{x}, t)\right]$,

where $a$ is the lattice spacing and $U_{4}(x)$ is the temporal gauge link operator. $H=H_{0}+\delta H$ is the NRQCD Hamiltonian, where

$$
H_{0}=-\frac{\tilde{\Delta}^{2}}{2 m_{b}}-\frac{a}{4 n} \frac{\left(\Delta^{2}\right)^{2}}{4 m_{b}^{2}} \quad \text { and } \quad \delta H=\sum_{i} \delta H^{(i)} .
$$

The $H_{0}$ is the leading $\mathcal{O}\left(v^{2}\right)$ term; the $\mathcal{O}\left(v^{4}\right)$ and $\mathcal{O}\left(v^{6}\right)$ terms are in $\delta H$ with coefficients $c_{1}$ through $c_{7}$,

$$
\begin{aligned}
& \delta H^{(1)}=-c_{1} \frac{\left(\Delta^{2}\right)^{2}}{8 m_{b}^{3}} \\
& \delta H^{(2)}=c_{2} \frac{i g}{8 m_{b}^{2}}\left(\vec{\Delta}^{ \pm} \cdot \vec{E}-\vec{E} \cdot \vec{\Delta}^{ \pm}\right) \\
& \delta H^{(3)}=-c_{3} \frac{g}{8 m_{b}^{2}} \vec{\sigma} \cdot\left(\tilde{\vec{\Delta}}^{ \pm} \times \tilde{\vec{E}}-\tilde{\vec{E}} \times \tilde{\vec{\Delta}}^{ \pm}\right) \\
& \delta H^{(4)}=-c_{4} \frac{g}{2 m_{b}} \vec{\sigma} \cdot \tilde{\vec{B}} \\
& \delta H^{(5)}=-c_{5} \frac{g}{8 m_{b}^{3}}\left\{\Delta^{2}, \vec{\sigma} \cdot \vec{B}\right\} \\
& \delta H^{(6)}=-c_{6} \frac{3 g}{64 m_{b}^{4}}\left\{\Delta^{2}, \vec{\sigma} \cdot\left(\vec{\Delta}^{ \pm} \times \vec{E}-\vec{E} \times \vec{\Delta}^{ \pm}\right)\right\} \\
& \delta H^{(7)}=-c_{7} \frac{i g^{2}}{8 m_{b}^{3}} \vec{\sigma} \cdot \vec{E} \times \vec{E} .
\end{aligned}
$$

The $b$ quark propagator is generated by the time evolution of this Hamiltonian,

$$
\begin{aligned}
G(\vec{x}, t+1 ; 0,0)= & \left(1-\frac{a H_{0}}{2 n}\right)^{n}\left(1-\frac{a \delta H}{2}\right) U_{4}(\vec{x}, t)^{\dagger} \\
& \times\left(1-\frac{a \delta H}{2}\right)\left(1-\frac{a H_{0}}{2 n}\right)^{n} G(\vec{x}, t ; 0,0)
\end{aligned}
$$

with

$$
G(\vec{x}, t ; 0,0)=\left\{\begin{array}{ll}
0 & \text { for } t<0 \\
\delta_{\vec{x}, 0} & \text { for } t=0
\end{array} .\right.
$$

The tree level value of all the coefficients $c_{1}, c_{2}, c_{3}, c_{4}, c_{5}$, $c_{6}$, and $c_{7}$ is 1 . Here, $n$ is the factor introduced to ensure numerical stability at small $a m_{b}$ [13], where $n>3 / 2 m_{b}$. The symmetric derivative $\Delta^{ \pm}$and Laplacian $\Delta^{2}$ in terms of forward and backward covariant derivatives are

$$
\begin{aligned}
a \Delta_{\mu}^{+} \psi(x) & =U_{\mu}(x) \psi(x+a \hat{\mu})-\psi(x) \\
a \Delta_{\mu}^{-} \psi(x) & =\psi(x)-U_{\mu}^{\dagger}(x-a \hat{\mu}) \psi(x-a \hat{\mu}) \\
\Delta^{ \pm} & =\frac{1}{2}\left(\Delta^{+}+\Delta^{-}\right) \\
\Delta^{2} & =\sum_{i} \Delta_{i}^{+} \Delta_{i}^{-}=\sum_{i} \Delta_{i}^{-} \Delta_{i}^{+} .
\end{aligned}
$$

By Taylor expanding the symmetric derivative and the Laplacian operator, we can find their forms corrected up to $\mathcal{O}\left(a^{4}\right)$ [12] that are used in the above Eq. (3), 


$$
\begin{aligned}
& \tilde{\Delta}_{i}^{ \pm}=\Delta_{i}^{ \pm}-\frac{a^{2}}{6} \Delta_{i}^{+} \Delta_{i}^{ \pm} \Delta_{i}^{-} \\
& \tilde{\Delta}^{2}=\Delta^{2}-\frac{a^{2}}{12} \sum_{i}\left[\Delta_{i}^{+} \Delta_{i}^{-}\right]^{2} .
\end{aligned}
$$

In the same way, the gauge fields are improved to $\mathcal{O}\left(a^{4}\right)$ using cloverleaf plaquette,

$$
\begin{aligned}
a \Delta_{\rho}^{+} F_{\mu \nu}(x) & =U_{\rho}(x) F_{\mu \nu}(x+a \hat{\rho}) U_{\rho}^{\dagger}(x)-F_{\mu \nu}(x) \\
a \Delta_{\rho}^{-} F_{\mu \nu}(x) & =F_{\mu \nu}(x)-U_{\rho}^{\dagger}(x-a \hat{\rho}) F_{\mu \nu}(x) U_{\rho}(x-a \hat{\rho}) \\
g \tilde{F}_{\mu \nu}(x) & =g F_{\mu \nu}(x)-\frac{a^{4}}{6}\left[\Delta_{\mu}^{+} \Delta_{\mu}^{-}+\Delta_{\nu}^{+} \Delta_{\nu}^{-}\right] g F_{\mu \nu}(x) .
\end{aligned}
$$

The chromoelectric $\tilde{E}$ and chromomagnetic $\tilde{B}$ fields in $\delta H^{(3)}$ and $\delta H^{(4)}$ of Eq. (3) are thus $\mathcal{O}\left(a^{4}\right)$ improved.

\section{B. HISQ charm and lighter quarks}

For the lighter quarks - charm, strange, and up/downrelativistic HISQ action [14] is used. Apart from anything else, from a practical point of coding the bottom-light operators $\left(l b b, l l b, l_{1} l_{2} b\right)$, using the same relativistic action for all lighter quarks offers a great degree of simplification. The HISQ action is given by

$$
\mathcal{S}=\sum_{x} \bar{l}(x)\left(\gamma^{\mu} D_{\mu}^{\mathrm{HISQ}}+m\right) l(x),
$$

where

$$
D_{\mu}^{\mathrm{HISQ}}=\Delta_{\mu}(W)-\frac{a^{2}}{6}(1+\epsilon) \Delta_{\mu}^{3}(X)
$$

with $W_{\mu}(x)=F_{\mu}^{\mathrm{HISQ}} U_{\mu}(x)$ and $X_{\mu}(x)=\mathcal{U} F_{\mu} U_{\mu}(x)$. The $F_{\mu}^{\mathrm{HISQ}}$ has the form,

$$
F_{\mu}^{\mathrm{HISQ}}=\left(F_{\mu}-\sum_{\rho \neq \mu} \frac{a^{2}\left(\delta_{\rho}\right)^{2}}{2}\right) \mathcal{U} F_{\mu} .
$$

Here, the $\mathcal{U}$ is the unitarizing operator, it unitarizes whatever it acts on, and the smearing operator $F_{\mu}$ is given by

$$
F_{\mu}=\prod_{\rho \neq \mu}\left(1+\frac{a^{2} \delta_{\rho}^{(2)}}{4}\right) .
$$

The $\delta_{\rho}$ and $\delta_{\rho}^{(2)}$ in the Eqs. (10) and (11) are covariant first and second order derivatives. Because HISQ action reduces $\mathcal{O}\left(\alpha_{s} a^{2}\right)$ discretization errors found in Asqtad action, it is well suited for $s$ and $u / d$ quarks. The parameter $\epsilon$ in the coefficient of the Naik term can be appropriately tuned to use the action for $c$ quarks. For $s$ and $u / d$ quarks, the $\epsilon=0$. Later, in the Table IV, we listed the parameters used for HISQ quarks. We have taken the values of $\epsilon$ from [16] and used MILC subroutines for generating HISQ propagators.
Since HISQ action is diagonal in spin space, propagators obtained do not have any spin structure. The full $4 \times 4$ spin structure can be regained by multiplying the propagators by the Kawamoto-Smit multiplicative phase factor [18],

$$
\Omega(x)=\prod_{\mu=1}^{4}\left(\gamma_{\mu}\right)^{x_{\mu}}=\gamma_{1}^{x_{1}} \gamma_{2}^{x_{2}} \gamma_{3}^{x_{3}} \gamma_{4}^{x_{4}} .
$$

MILC library uses a different representation of $\gamma$ matrices than the ones used in NRQCD. However, $\gamma$ matrices of these two representations are related by the unitary transformation of the form,

$S \gamma_{\mu}^{\mathrm{MILC}} S^{\dagger}=\gamma_{\mu}^{\mathrm{NR}} \quad$ where, $S=\frac{1}{\sqrt{2}}\left(\begin{array}{cc}\sigma_{y} & \sigma_{y} \\ -\sigma_{y} & \sigma_{y}\end{array}\right)$.

\section{TWO-POINT FUNCTIONS}

In this section, we discuss the construction of the bottom baryons by combining spin and color indices of the appropriate quark fields to form necessary baryon operators and two-point functions. The $b$ quark field is universally represented with $Q$ throughout the paper. It is defined later in the Eq. (17).

\section{A. Bottom-bottom meson two-point function}

After the $b$ quark propagators are generated according to Eq. (4), we calculate the masses of bottomonium states from the exponential falloff of two-point functions, i.e., correlators of the state with quantum numbers of interest. The meson creation operators are constructed from two component quark and antiquark creation operators $\psi_{h}^{\dagger}$ and $\chi_{h}^{\dagger}[19,20]$. As antiquarks transform as $\overline{3}$ under color rotation, we rename the antiquark spinor as $\chi_{h} \equiv \chi_{h}^{*}$ [13]. The meson creation operator is, thus,

$$
\mathcal{O}_{h h}(x)=\psi_{h}^{\dagger}(x) \Gamma \chi_{h}(x) .
$$

Heavy-heavy, i.e., bottom-bottom meson two-point function is then given by $[13,21]$

$$
\begin{aligned}
C_{h h}(\vec{p}, t) & =\sum_{\vec{x}}\left\langle\mathcal{O}_{h h}^{\dagger}(x) \mathcal{O}_{h h}(0)\right\rangle \\
& =\sum_{\vec{x}} e^{i \vec{p} \cdot \vec{x}} \operatorname{Tr}\left[G^{\dagger}(x, 0) \Gamma_{\text {sink }}^{\dagger} G(x, 0) \Gamma_{\text {src }}\right],
\end{aligned}
$$

$\Gamma_{\text {sink }}=\Gamma_{\text {src }}=I$ and $\sigma_{i}$ for the pseudoscalar and vector mesons, respectively. The heavy-heavy propagator $G(x, 0)$ is a $2 \times 2$ matrix in spin space. If we think of $G(x, 0)$ as a $4 \times 4$ matrix with vanishing lower components then we can rewrite the above Eq. (15) as [9]

$$
C_{h h}(\vec{p}, t)=\sum_{\vec{x}} e^{i \vec{p} \cdot \vec{x}} \operatorname{Tr}\left[\gamma_{5} G^{\dagger}(x, 0) \gamma_{5} \Gamma_{\text {sink }}^{\dagger} G(x, 0) \Gamma_{\text {src }}\right],
$$


where $\Gamma$ matrices now changed to $\Gamma_{\text {sink }}=\Gamma_{\text {src }}=\gamma_{5}$ and $\gamma_{i}$ for pseudoscalar and vector mesons, respectively. In Eq. (16), we have used the nonrelativistic Dirac representation of $\gamma$ matrices. In the Eqs. (15), (16), and (19), the trace is taken over both the spin and color indices.

\section{B. Heavy-light meson two-point function}

As discussed above, $b$ quark field $\psi_{h}$ has only two spin components. We convert it to a four-component spinor having vanishing lower components,

$$
Q=\left(\begin{array}{c}
\psi_{h} \\
0
\end{array}\right) \text {. }
$$

This helps us to combine the $b$ and light quark fields in the usual way,

$$
\mathcal{O}_{h l}(x)=\bar{Q}(x) \Gamma l(x),
$$

where $l(x)$ stands for the light quark fields, $\bar{Q}=Q^{\dagger} \gamma_{4}$, and depending on pseudoscalar and vector mesons $\Gamma=\gamma_{5}$ and $\gamma_{i}$, respectively. Note that in the Dirac, i.e., the NR representation of $\gamma$ matrices $\gamma_{4} Q=Q$. The zero momentum bottom-light two-point function becomes [10,22]

$$
\begin{aligned}
C_{h l}(t) & =\sum_{\vec{x}}\left\langle\mathcal{O}_{h l}^{\dagger}(x) \mathcal{O}_{h l}(0)\right\rangle \\
& =\sum_{\vec{x}} \operatorname{Tr}\left[\gamma_{5} M^{\dagger}(x, 0) \gamma_{5} \Gamma_{\text {sink }}^{\dagger} G(x, 0) \Gamma_{\text {src }}\right],
\end{aligned}
$$

where $M(x, 0)$ is the light quark propagator. It has the usual full $4 \times 4$ spin structure. As before, $G(x, 0)$ is the $b$ quark propagator having vanishing lower components. However, before implementing Eq. (19), $G(x, 0)$ has to be rotated to the MILC basis.

\section{Bottom baryon two-point functions}

The bottom quark field $Q$ has vanishing lower components and hence, can be projected to positive parity states only. Besides, the use of $\Gamma=C \gamma_{5}$ in a diquark operator made from same flavor, i.e., $l^{T} C \gamma_{5} l$ is not allowed by the Pauli exclusion principle. In other words, the insertion of $C \gamma_{5}$ between two quark fields of the same flavor creates a combination which is antisymmetric in spin indices, while the presence of $\epsilon_{a b c}$ makes the combination antisymmetric in color indices. This makes the overall operator become symmetric under the interchange of the same flavored quark fields. Keeping this in mind, the constructions of various bottom baryon two-point functions are described below.

\section{Triply bottom baryon}

A triply bottom baryon operator is defined by

$$
\left(\mathcal{O}_{k}^{h h h}\right)_{\alpha}=\epsilon_{a b c}\left(Q^{a T} C \gamma_{k} Q^{b}\right) Q_{\alpha}^{c},
$$

where $C=\gamma_{4} \gamma_{2}$. Here, $a, b, c$ are the color indices, $\alpha$ is the spinor index, and $k$ is the Lorentz index, which runs from 1 to 3 . The zero momentum two-point function reads [9]

$$
\begin{aligned}
C_{j k ; \alpha \delta}^{h h h}(t)= & \sum_{\vec{x}}\left\langle\left[\mathcal{O}_{j}^{h h h}(x)\right]_{\alpha}\left[\mathcal{O}_{k}^{h h h}(0)\right]_{\delta}^{\dagger}\right\rangle \\
= & \sum_{\vec{x}} \epsilon_{a b c} \epsilon_{f g h} G_{\alpha \delta}^{c h}(x, 0) \\
& \times \operatorname{Tr}\left[C \gamma_{j} G^{b g}(x, 0) \gamma_{k} \gamma_{2} G^{a f T}(x, 0)\right] .
\end{aligned}
$$

In the above Eq. (21) and the subsequent ones, the transpose and traces are taken over spin indices. Baryon operators having $C \gamma_{k}$ in the diquark component have overlap with both spin- $\frac{3}{2}$ and $\frac{1}{2}$ states. For example, the correlator defined in Eq. (21) can be written explicitly as an overlap with both spin- $\frac{3}{2}$ and $\frac{1}{2}$ states [23],

$$
C_{i j}^{h h h}(t)=Z_{3 / 2}^{2} e^{-E_{3 / 2} t} \Pi P_{i j}^{3 / 2}+Z_{1 / 2}^{2} e^{-E_{1 / 2} t} \Pi P_{i j}^{1 / 2},
$$

where $\Pi=\left(1+\gamma_{4}\right) / 2$ and the spin projection operators $P_{i j}^{3 / 2}=\delta_{i j}-\gamma_{i} \gamma_{j} / 3$ and $P_{i j}^{1 / 2}=\gamma_{i} \gamma_{j} / 3$. The individual contribution to the respective spin states can be obtained by taking appropriate projections,

$$
\begin{aligned}
& P_{i j}^{3 / 2} C_{j k}^{h h h}=Z_{3 / 2}^{2} \Pi e^{-E_{3 / 2} t} P_{i k}^{3 / 2} \\
& P_{i j}^{1 / 2} C_{j k}^{h h h}=Z_{1 / 2}^{2} \Pi e^{-E_{1 / 2} t} P_{i k}^{1 / 2} .
\end{aligned}
$$

In this paper, we use these projections to separate the different spin states. We would like to point out that the spin $-\frac{1}{2}$ state of triply bottom baryon is not a physical state as it violates Pauli exclusion principle even though we can take the projection in practice.

\section{Bottom-bottom-light baryon}

Interpolating operator for baryons, having two $b$ quarks and a light quark, can be constructed in two ways based on how the diquark component is formed [24],

$$
\begin{aligned}
& \left(\mathcal{O}_{k}^{h h l}\right)_{\alpha}=\epsilon_{a b c}\left(Q^{a T} C \gamma_{k} Q^{b}\right) l_{\alpha}^{c} \\
& \left(\mathcal{O}_{k}^{h l h}\right)_{\alpha}=\epsilon_{a b c}\left(Q^{a T} C \gamma_{k} l^{b}\right) Q_{\alpha}^{c} .
\end{aligned}
$$

The corresponding baryon correlators are

$$
\begin{aligned}
C_{j k ; \alpha \delta}^{h h l}(t)= & \sum_{\vec{x}}\left\langle\left[\mathcal{O}_{j}^{h h l}(x)\right]_{\alpha}\left[\mathcal{O}_{k}^{h h l}(0)\right]_{\delta}^{\dagger}\right\rangle \\
= & \sum_{\vec{x}} \epsilon_{a b c} \epsilon_{f g h}\left[M^{c h}(x, 0) \gamma_{4}\right]_{\alpha \delta} \\
& \times \operatorname{Tr}\left[\gamma_{4} \gamma_{2} \gamma_{j} G^{b g}(x, 0) \gamma_{k} \gamma_{2} G^{a f T}(x, 0)\right]
\end{aligned}
$$




$$
\begin{aligned}
C_{j k ; \alpha \delta}^{h l h}(t)= & \sum_{\vec{x}}\left\langle\left[\mathcal{O}_{j}^{h l h}(x)\right]_{\alpha}\left[\mathcal{O}_{k}^{h l h}(0)\right]_{\delta}^{\dagger}\right\rangle \\
= & \sum_{\vec{x}} \epsilon_{a b c} \epsilon_{f g h} G_{\alpha \delta}^{c h}(x, 0) \\
& \times \operatorname{Tr}\left[\gamma_{4} \gamma_{2} \gamma_{j} M^{b g}(x, 0) \gamma_{k} \gamma_{2} G^{a f T}(x, 0)\right] .
\end{aligned}
$$

The propagator $G(x, 0)$ is required to be converted to the MILC basis using the unitary matrix $S$ defined in Eq. (13).

An additional spin- $-\frac{1}{2}$ operator can be defined for the $\mathcal{O}^{\text {hlh }}$ type operator as

$$
\left(\mathcal{O}_{5}^{h l h}\right)_{\alpha}=\epsilon_{a b c}\left(Q^{a T} C \gamma_{5} l^{b}\right) Q_{\alpha}^{c} .
$$

The two-point function for this operator is obtained by replacing $\gamma_{j}$ and $\gamma_{k}$ by $\gamma_{5}$ in Eq. (27). We cannot have a $C \gamma_{5}$ between two $Q$ in diquark and hence, no $\mathcal{O}_{5}^{h h l}$.

In Table I, we tabulated the full list of triple and double bottom baryon operators that are used in this work. We have broadly followed the nomenclature adopted in [11] but with certain modifications as needed for this work. The baryons having the same quark content and $J^{P}$ are obtained in two different ways, as mentioned above. The operators with "tilde", for instance, $\tilde{\Omega}_{b b}\left(1 / 2^{+}\right)$, are obtained by projecting the relevant $\left(Q C \gamma_{k} l\right) Q$ operator with $P_{i j}^{1 / 2}$. The operators with "prime", such as $\Omega_{b b}^{\prime}\left(1 / 2^{+}\right)$, are obtained from $\left(Q C \gamma_{5} l\right) Q$ diquark construction. The "prime" states so constructed on lattice correspond to the "prime" continuum states, such as $\Xi_{b}^{\prime}$ or till unobserved $\Omega_{c b}^{\prime}$ etc. It is obvious that baryon states calculated by projecting out definite spin states from a two-point function share the same interpolating operator. The starred baryons are for $J^{P}=3 / 2^{+}$states.

\section{Bottom-light-light baryon}

The natural choice for an interpolating operator, as motivated by the Heavy Quark Effective Theory (HQET) [25], in $l l h$-baryon kind is

TABLE I. Operators for triple and double bottom baryons. $Q$ is used for $b$ field and $l$ for any of the $c, s, u / d$ lighter quarks.

\begin{tabular}{lccc}
\hline \hline Baryon & Quark content & $J^{P}$ & Operator \\
\hline$\Omega_{b b b}$ & $b b b$ & $\frac{3}{2}^{+}, \frac{1}{2}^{+}$ & $\epsilon_{a b c}\left(Q^{a T} C \gamma_{k} Q^{b}\right) Q^{c}$ \\
$\Omega_{c b b}^{\star}, \Omega_{c b b}$ & $c b b$ & $\frac{3}{2}^{+}, \frac{1}{2}^{+}$ & $\epsilon_{a b c}\left(Q^{a T} C \gamma_{k} Q^{b}\right) l^{c}$ \\
$\tilde{\Omega}_{c b b}^{\star}, \tilde{\Omega}_{c b b}$ & $c b b$ & $\frac{3}{2}^{+}, \frac{1}{2}^{+}$ & $\epsilon_{a b c}\left(Q^{a T} C \gamma_{k} l^{b}\right) Q^{c}$ \\
$\Omega_{c b b}^{\prime}$ & $c b b$ & $\frac{1}{2}^{+}$ & $\epsilon_{a b c}\left(Q^{a T} C \gamma_{5} l^{b}\right) Q^{c}$ \\
$\Omega_{b b}^{\star}, \Omega_{b b}$ & $s b b$ & $\frac{3}{2}^{+}, \frac{1}{2}+$ & $\epsilon_{a b c}\left(Q^{a T} C \gamma_{k} Q^{b}\right) l^{c}$ \\
$\tilde{\Omega}_{b b}^{\star}, \tilde{\Omega}_{b b}$ & $s b b$ & $\frac{3}{2}^{+}, \frac{1}{2}+$ & $\epsilon_{a b c}\left(Q^{a T} C \gamma_{k} l^{b}\right) Q^{c}$ \\
$\Omega_{b b}^{\prime}$ & $s b b$ & $\frac{1}{2}+$ & $\epsilon_{a b c}\left(Q^{a T} C \gamma_{5} l^{b}\right) Q^{c}$ \\
$\Xi_{b b}^{\star}, \Xi_{b b}$ & $u b b$ & $\frac{3}{2}+, \frac{1}{2}+$ & $\epsilon_{a b c}\left(Q^{a T} C \gamma_{k} Q^{b}\right) l^{c}$ \\
$\tilde{\Xi}_{b b}^{\star}, \tilde{\Xi}_{b b}$ & $u b b$ & $\frac{3}{2}+, \frac{1}{2}+$ & $\epsilon_{a b c}\left(Q^{a T} C \gamma_{k} l^{b}\right) Q^{c}$ \\
$\Xi_{b b}^{\prime}$ & $u b b$ & $\frac{1}{2}{ }^{+}$ & $\epsilon_{a b c}\left(Q^{a T} C \gamma_{5} l^{b}\right) Q^{c}$ \\
\hline \hline
\end{tabular}

$$
\left(\mathcal{O}_{k}^{h l_{1} l_{2}}\right)_{\alpha}=\epsilon_{a b c}\left(l_{1}^{a T} C \gamma_{k} l_{2}^{b}\right) Q_{\alpha}^{c},
$$

and the corresponding two-point function is

$$
\begin{aligned}
C_{j k ; \delta \delta}^{h l_{1} l_{2}}(t)= & \sum_{\vec{x}}\left\langle\left[\mathcal{O}_{j}^{h l_{1} l_{2}}(x)\right]_{\alpha}\left[\mathcal{O}_{k}^{h l_{1} l_{2}}(0)\right]_{\delta}^{\dagger}\right\rangle \\
= & \sum_{\vec{x}} \epsilon_{a b c} \epsilon_{f g h} G_{\alpha \delta}^{c h}(x, 0) \\
& \times \operatorname{Tr}\left[\gamma_{4} \gamma_{2} \gamma_{j} M_{2}^{b g}(x, 0) \gamma_{k} \gamma_{2} \gamma_{4} M_{1}^{a f T}(x, 0)\right] .
\end{aligned}
$$

In HISQ formalism for light quarks, the corresponding propagators $M_{1}(x, 0)$ and $M_{2}(x, 0)$ have the same Kawamoto-Smit multiplicative factor $\Omega(x)$. Hence, they have the same spin structure irrespective of color indices. As a result, the trace over spin indices in Eq. (30) vanishes if $\gamma_{j} \neq \gamma_{k}$. Therefore, we can not separate the two spin- $\frac{3}{2}$ and $\frac{1}{2}$ states. If we want to use the same diquark structure as in Eq. (29), for baryons having different light quark flavors, we can define the spin $-\frac{1}{2}$ operator by

$$
\left(\mathcal{O}_{5}^{h l_{1} l_{2}}\right)_{\alpha}=\epsilon_{a b c}\left(l_{1}^{a T} C \gamma_{5} l_{2}^{b}\right) Q_{\alpha}^{c}
$$

and the corresponding two-point function is

$$
\begin{aligned}
C_{55 ; \alpha \delta}^{h l_{1} l_{2}}(t)= & \sum_{\vec{x}}\left\langle\left[\mathcal{O}_{5}^{h l_{1} l_{2}}(x)\right]_{\alpha}\left[\mathcal{O}_{5}^{h l_{1} l_{2}}(0)\right]_{\delta}^{\dagger}\right\rangle \\
= & \sum_{\vec{x}} \epsilon_{a b c} \epsilon_{f g h} G_{\alpha \delta}^{c h}(x, 0) \\
& \times \operatorname{Tr}\left[\gamma_{4} \gamma_{2} \gamma_{5} M_{2}^{b g}(x, 0) \gamma_{5} \gamma_{2} \gamma_{4} M_{1}^{a f T}(x, 0)\right] .
\end{aligned}
$$

Besides, instead of Eq. (29), we can choose our $\left(h l_{1} l_{2}\right)$ operator as

$$
\left(\mathcal{O}_{k}^{h l_{2} l_{1}}\right)_{\alpha}=\epsilon_{a b c}\left(Q^{a T} C \gamma_{k} l_{2}^{b}\right) l_{1 \alpha}^{c}
$$

The two-point function is now

$$
\begin{aligned}
C_{j k, \alpha \delta}^{h l_{2} l_{1}}(t)= & \sum_{\vec{x}} \epsilon_{a b c} \epsilon_{f g h}\left[M_{1}^{c h}(x, 0) \gamma_{4}\right]_{\alpha \delta} \\
& \times \operatorname{Tr}\left[\gamma_{4} \gamma_{2} \gamma_{j} M_{2}^{b g}(x, 0) \gamma_{k} \gamma_{2} G^{a f T}(x, 0)\right] .
\end{aligned}
$$

Because the light quark propagators $M_{1}(x, 0)$ and $M_{2}(x, 0)$ are proportional to each other, the relative positions of the quark fields $l_{1}$ and $l_{2}$ in Eq. (33) is irrelevant. The interpolating operator defined in Eq. (33) has overlap with both spin- $-\frac{3}{2}$ and $\frac{1}{2}$ states and can be projected out by appropriate projection operators $P_{i j}^{1 / 2,3 / 2}$.

As before, we can also define an additional spin $-\frac{1}{2}$ operator here too,

$$
\left(\mathcal{O}_{5}^{h l_{2} l_{1}}\right)_{\alpha}=\epsilon_{a b c}\left(Q^{a T} C \gamma_{5} l_{2}^{b}\right) l_{1 \alpha}^{c} .
$$

The two-point function for this operator has the same form as in Eq. (34) with $\gamma_{j}$ and $\gamma_{k}$ replaced by $\gamma_{5}$. 
TABLE II. Operators for single bottom baryons. $Q$ is used for $b$ field as before. Interchange in the position of two lighter quarks keeps the operator unchanged.

\begin{tabular}{lccc}
\hline \hline Baryon & Quark content & $J^{P}$ & Operator \\
\hline$\tilde{\Omega}_{c c b}^{\star}, \tilde{\Omega}_{c c b}$ & $c c b$ & $\frac{3}{2}^{+}, \frac{1}{2}{ }^{+}$ & $\epsilon_{a b c}\left(Q^{a T} C \gamma_{k} c^{b}\right) c^{c}$ \\
$\Omega_{c c b}^{\prime}$ & $c c b$ & $\frac{1}{2}^{+}$ & $\epsilon_{a b c}\left(Q^{a T} C \gamma_{5} c^{b}\right) c^{c}$ \\
$\Omega_{c b}$ & $s c b$ & $\frac{1}{2}^{+}$ & $\epsilon_{a b c}\left(s^{a T} C \gamma_{5} c^{b}\right) Q^{c}$ \\
$\tilde{\Omega}_{c b}^{\star}, \tilde{\Omega}_{c b}$ & $s c b$ & $\frac{3}{2}^{+}, \frac{1}{2}{ }^{+}$ & $\epsilon_{a b c}\left(Q^{a T} C \gamma_{k} c^{b}\right) s^{c}$ \\
$\Omega_{c b}^{\prime}$ & $s c b$ & $\frac{1}{2}{ }^{+}$ & $\epsilon_{a b c}\left(Q^{a T} C \gamma_{5} c^{b}\right) s^{c}$ \\
$\Xi_{c b}$ & $u c b$ & $\frac{1}{2}{ }^{+}$ & $\epsilon_{a b c}\left(u^{a T} C \gamma_{5} c^{b}\right) Q^{c}$ \\
$\tilde{\Xi}_{c b}^{\star}, \tilde{\Xi}_{c b}$ & $u c b$ & $\frac{3}{2}{ }^{+}, \frac{1}{2}{ }^{+}$ & $\epsilon_{a b c}\left(Q^{a T} C \gamma_{k} c^{b}\right) u^{c}$ \\
$\Xi_{c b}^{\prime}$ & $u c b$ & $\frac{1}{2}{ }^{+}$ & $\epsilon_{a b c}\left(Q^{a T} C \gamma_{5} c^{b}\right) u^{c}$ \\
$\tilde{\Omega}_{b}^{\star}, \tilde{\Omega}_{b}$ & $s s b$ & $\frac{3}{2}+, \frac{1}{2}{ }^{+}$ & $\epsilon_{a b c}\left(Q^{a T} C \gamma_{k} s^{b}\right) s^{c}$ \\
$\Omega_{b}^{\prime}$ & $s s b$ & $\frac{1}{2}{ }^{+}$ & $\epsilon_{a b c}\left(Q^{a T} C \gamma_{5} s^{b}\right) s^{c}$ \\
$\Xi_{b}$ & $u s b$ & $\frac{1}{2}{ }^{+}$ & $\epsilon_{a b c}\left(u^{a T} C \gamma_{5} s^{b}\right) Q^{c}$ \\
$\tilde{\Xi}_{b}^{\star}, \tilde{\Xi}_{b}$ & $u s b$ & $\frac{3}{2}{ }^{+}, \frac{1}{2}{ }^{+}$ & $\epsilon_{a b c}\left(Q^{a T} C \gamma_{k} s^{b}\right) u^{c}$ \\
$\Xi_{b}^{\prime}$ & $u s b$ & $\frac{1}{2}{ }^{+}$ & $\epsilon_{a b c}\left(Q^{a T} C \gamma_{5} s^{b}\right) u^{c}$ \\
$\tilde{\Sigma}_{b}^{\star}, \tilde{\Sigma}_{b}$ & $u u b$ & $\frac{3}{2}{ }^{+}, \frac{1}{2}{ }^{+}$ & $\epsilon_{a b c}\left(Q^{a T} C \gamma_{k} u^{b}\right) u^{c}$ \\
$\Sigma_{b}^{\prime}$ & $u u b$ & $\frac{1}{2}{ }^{+}$ & $\epsilon_{a b c}\left(Q^{a T} C \gamma_{5} u^{b}\right) u^{c}$ \\
$\Lambda_{b}$ & $u d b$ & $\frac{1}{2}{ }^{+}$ & $\epsilon_{a b c}\left(u^{a T} C \gamma_{5} d^{b}\right) Q^{c}$ \\
\hline \hline
\end{tabular}

In Table II, we tabulate our full list of single bottom baryon operators that we made use of in this work. The "tilde" and "prime" states that appear in the table have been explained before in the context of multi bottom baryon operators.

\section{Light baryon}

We occasionally need charmed baryon states like $q c c$ or $q q c$, where $q$ is any of $s$ or $u / d$ quarks or both; hence, we include a discussion on charmed baryon operators. The $c$ quark in the present case is relativistic. For the reason discussed above, with HISQ action for lighter quarks we can define only the spin- $\frac{1}{2}$ operators. Consider a $\left(l_{1} l_{2} l_{3}\right)$-baryon where at least two quarks are differently flavored, say $l_{1} \neq l_{2}$. The spin- $\frac{1}{2}$ operator and the corresponding two-point function in such case is

$$
\begin{gathered}
\left(\mathcal{O}_{5}^{l_{1} l_{2} l_{3}}\right)_{\alpha}=\epsilon_{a b c}\left(l_{1}^{a T} C \gamma_{5} l_{2}^{b}\right) l_{3 \alpha}^{c} \\
C_{55, \alpha \delta}^{l_{1} l_{2} l_{3}}(t)=\sum_{\vec{x}} \epsilon_{a b c} \epsilon_{f g h}\left[M_{3}^{c h}(x, 0) \gamma_{4}\right]_{\alpha \delta} \\
\times \operatorname{Tr}\left[\gamma_{4} \gamma_{2} \gamma_{5} M_{2}^{b g}(x, 0) \gamma_{5} \gamma_{2} \gamma_{4} M_{1}^{a f T}(x, 0)\right] .
\end{gathered}
$$

The two light baryon states $\left(J^{P}=1 / 2^{+}\right)$that we are interested in this work are

$$
\begin{aligned}
& \Sigma_{c}(u u c): \epsilon_{a b c}\left(c^{a T} C \gamma_{5} u^{b}\right) u^{c} \\
& \Xi_{c c}(u c c): \epsilon_{a b c}\left(c^{a T} C \gamma_{5} u^{b}\right) c^{c} .
\end{aligned}
$$

\section{SIMULATION DETAILS}

We calculated the bottom baryon spectra using the publicly available $N_{f}=2+1$ Asqtad gauge configurations generated by the MILC Collaboration. Details about these lattices can be found in [26]. It uses Symanzikimproved Lüscher-Weisz action for the gluons and Asqtad action $[27,28]$ for the sea quarks. The lattices we choose have a fixed ratio of $a m_{l} / a m_{s}=1 / 5$ and lattice spacings ranging from 0.15 to $0.09 \mathrm{fm}$ corresponding to the same physical volume. We have not determined the lattice spacings independently but use those given in [26]. In Table III, we listed the ensembles used in this work.

In NRQCD, the rest mass term does not appear in Eq. (3), and therefore, we cannot determine hadron masses from their energies at zero momentum directly from the exponential falloff of the correlation functions. Instead, we calculate the kinetic mass $M_{k}$ of heavy-heavy mesons from its energy-momentum relation, which to $\mathcal{O}\left(p^{2}\right)$ is [15],

$$
\begin{aligned}
E(p) & =E(0)+\sqrt{p^{2}+M_{k}^{2}}-M_{k} \\
\Rightarrow \quad E(p)^{2} & =E(0)^{2}+\frac{E(0)}{M_{k}} p^{2} .
\end{aligned}
$$

We calculate the $E(p)$ at different values of lattice momenta $p=2 n \pi / L$, where, $n=(0,0,0),(1,0,0),(1,1,0),(1,1,1)$, $(2,0,0),(2,1,0)$, and $(2,1,1)$.

\section{A. $m_{b}$ tuning}

The $b$ quark mass is tuned from the spin average $\Upsilon$ and $\eta_{b}$ masses,

$$
M_{b \bar{b}}=\frac{3 M_{\Upsilon}+M_{\eta_{b}}}{4},
$$

using kinetic mass for both $\Upsilon$ and $\eta_{b}$. The experimental value to which $M_{b \bar{b}}$ is tuned to is not $9443 \mathrm{MeV}$, as obtained from spin averaging $\Upsilon(9460 \mathrm{MeV})$ and $\eta_{b}$ (9391 MeV) experimental masses, but to an appropriately adjusted value of $9450 \mathrm{MeV}$ [16], which we denote as $M_{\text {phys }}^{\text {mod }}$ later in the Eq. (41). The reasons being, firstly, electromagnetic interaction among the quarks are not considered here. Secondly, the disconnected diagrams while computing a two-point function are also not considered thus, not allowing $b, \bar{b}$ quarks to annihilate to gluons. And

TABLE III. MILC configurations used in this work. The gauge coupling is $\beta$, lattice spacing $a, u / d$ and $s$ sea quark masses are $m_{l}$ and $m_{s}$, respectively, and lattice size is $L^{3} \times T$. The $N_{\mathrm{cfg}}$ is the number of configurations used in this work.

\begin{tabular}{lcllcl}
\hline \hline$\beta=10 / g^{2}$ & $a(\mathrm{fm})$ & \multicolumn{1}{c}{$a m_{l}$} & \multicolumn{1}{c}{$a m_{s}$} & $L^{3} \times T$ & $N_{\text {cfg }}$ \\
\hline 6.572 & 0.15 & 0.0097 & 0.0484 & $16^{3} \times 48$ & 400 \\
6.76 & 0.12 & 0.01 & 0.05 & $20^{3} \times 64$ & 400 \\
7.09 & 0.09 & 0.0062 & 0.031 & $28^{3} \times 96$ & 300 \\
\hline \hline
\end{tabular}


TABLE IV. Tuned $b, c$, and $s$ quark bare masses for lattices used in this work. For the $s$-quark mass, we mentioned the particle states to which it is tuned to. The values of $\epsilon$ parameter used for the $c$ quark are given in the last column.

\begin{tabular}{lccccc}
\hline \hline$a(\mathrm{fm})$ & $a m_{b}$ & $a m_{c}$ & $a m_{s}\left(\eta_{s}\right)$ & $a m_{s}\left(B_{s}\right)$ & $\epsilon[16]$ \\
\hline 0.15 & 2.76 & 0.850 & 0.065 & 0.215 & -0.34 \\
0.12 & 2.08 & 0.632 & 0.049 & 0.155 & -0.21 \\
0.09 & 1.20 & 0.452 & 0.0385 & 0.114 & -0.115 \\
\hline \hline
\end{tabular}

finally, we do not have sea $c$ quarks in our simulation. For a more detailed discussion on this, see [16].

The $b$ quark mass $m_{b}$ and the coefficient $c_{4}$ in Eq. (3) are then tuned to obtain a modified spin average mass and the hyperfine splitting of $\Upsilon$ and $\eta_{b}$, which is $\sim 60-65 \mathrm{MeV}$ [29]. In order to achieve the desired hyperfine splittings, we tuned only $c_{4}$ since at $\mathcal{O}\left(1 / m_{b}\right)$, this is the only term that contains Pauli spin matrices. Therefore, it allows the mixing of spin components of $\psi_{h}$. This term contributes maximally to the hyperfine splitting compared to the others that contain Pauli spin matrices. The one-loop radiative correction to $c_{4}$ [30] has been used to tune hyperfine splitting in [31], where it was found to change, but only mildly, over lattice spacings $\sim 0.15-0.09 \mathrm{fm}$ for $N_{f}=$ $2+1+1$ HISQ gauge configurations. In our present mixed action study, the changes in the tuned $c_{4}$ on various lattice ensembles are small enough. Taking an average of those values, we choose $c_{4}=1.9$ for which the hyperfine splittings obtained on three different lattices $0.15,0.12$, and $0.09 \mathrm{fm}$ are $60.6,61.1$, and $61.8 \mathrm{MeV}$, respectively.

All other coefficients $c_{i}$ in Eq. (3) are set to 1.0. We set stability factor $n=4$ throughout our simulation. The Table IV lists the values of $m_{b}$ used in this work.

\section{B. $\boldsymbol{m}_{c}$ tuning}

The $c$-quark mass is tuned pretty much in the same way as $m_{b}$, except that $M_{c \bar{c}}$ is tuned to the spin average of $J / \psi$ and $\eta_{c}$ experimental masses. In this case, however, the adjustment to the spin averaged value due to the absence of electromagnetic interaction, $c$ quarks in sea, and disconnected diagrams are very small and hence, neglected. The bare $c$-quark masses used in this work are given in Table IV.

\section{C. $m_{s}$ tuning}

The $s$-quark mass is tuned to two different values. In the first case, we tune to the mass of fictitious $s \bar{s}$ pseudoscalar meson $\eta_{s}$ while in the second case, to the mass of $B_{s}$. The $\eta_{s}$ is a fictitious meson that is not allowed to decay through $s \bar{s}$ annihilation. Hence, no disconnected diagrams arise in the two-point function calculation. From chiral perturbation theory, its mass is estimated to be $m_{\eta_{s}}=\sqrt{2 m_{K}^{2}-m_{\pi}^{2}}=$ $689 \mathrm{MeV}[32,33]$. The $s$-quark mass thus tuned is checked against $D_{s}$ meson, making use of the $c$-quark mass obtained
TABLE V. $\quad D$ and $B$ meson masses in $\mathrm{MeV}$ with the tuned $a m_{b}$, $a m_{c}$, and $a m_{s}$.

\begin{tabular}{llll}
\hline \hline & & \multicolumn{2}{c}{$D_{s}$} \\
\cline { 3 - 4 }$L^{3} \times T$ & \multicolumn{1}{c}{$B_{c}$} & \multicolumn{1}{c}{$\eta_{s}$} & \multicolumn{1}{c}{$B_{s}$} \\
\hline $16^{3} \times 48$ & $6260(8)$ & $1994(3)$ & $2197(2)$ \\
$20^{3} \times 64$ & $6263(12)$ & $1977(4)$ & $2172(2)$ \\
$28^{3} \times 96$ & $6255(10)$ & $1971(3)$ & $2167(2)$ \\
PDG [8] & 6275 & 1968 & \\
\hline \hline
\end{tabular}

above and found to agree with the experimental $D_{s}$ (1968 MeV).

Next, we explore, tuning $m_{s}$ when a $s$ quark is in a bound state with a heavy $b$ quark. Here, we are assuming that the potential experienced by the $s$ quark in the field of a $b$ quark in a $B_{s}$ meson remains the same in other strange bottom baryons, and there is no spin-spin interactions taking place between the quarks. In the infinite mass limit, the HQET Lagrangian becomes invariant under arbitrary spin rotations [25]. Thereby, we can argue that for $s b b$ and $s c b$ systems the spin-spin interactions do not contribute significantly in spectrum calculation. However, this argument is perhaps not valid in systems like $b s s$ or $b s d$ but still with $s$ quark thus tuned; we possibly can obtain their masses close to their physical masses without resorting to any extrapolation.

In this paper, we will present our results obtained at these two different values of $m_{s}$. In Table IV, we listed these values of $s$-quark masses. In the Table $\mathrm{V}$, we calculate $B_{c}$ and $D_{s}$ mesons using tuned $b, c$, and $s$ masses. As is seen, when $m_{s}$ is tuned with $\eta_{s}$ the $D_{s}$ mass obtained is fairly close to the PDG value, whereas when tuned to $B_{s}$, we see an upward shift by an average $200 \mathrm{MeV}$. We have observed similar differences when $s$ quark appears together with $c$ in $(s c b)$-baryon masses.

\section{D. $u / d$ quarks}

For the valence $u / d$ quark mass, we used a range of bare quark masses varying from the lightest sea quark masses all the way to a little above, where the $s$ mass is tuned to $B_{s}$. Whenever the mass of a bottom baryon containing $u / d$ quark(s) is quoted, it will correspond to the $u / d$ quark mass tuned at the $B$ mass. Since we are not including either

TABLE VI. Values of $a m_{u / d}$ used in this work.

\begin{tabular}{lc}
\hline \hline$L^{3} \times T$ & $a m_{u / d}$ \\
\hline $16^{3} \times 48$ & $0.065,0.10,0.13,0.14,0.155$, \\
$(0.15 \mathrm{fm})$ & $0.165,0.185,0.215,0.225$ \\
$20^{3} \times 64$ & $0.05,0.075,0.090,0.10$, \\
$(0.12 \mathrm{fm})$ & $0.115,0.13,0.155,0.165$ \\
$28^{3} \times 96$ & $0.04,0.07,0.085,0.09$, \\
$(0.09 \mathrm{fm})$ & $0.10,0.114,0.12,0.13$ \\
\hline \hline
\end{tabular}




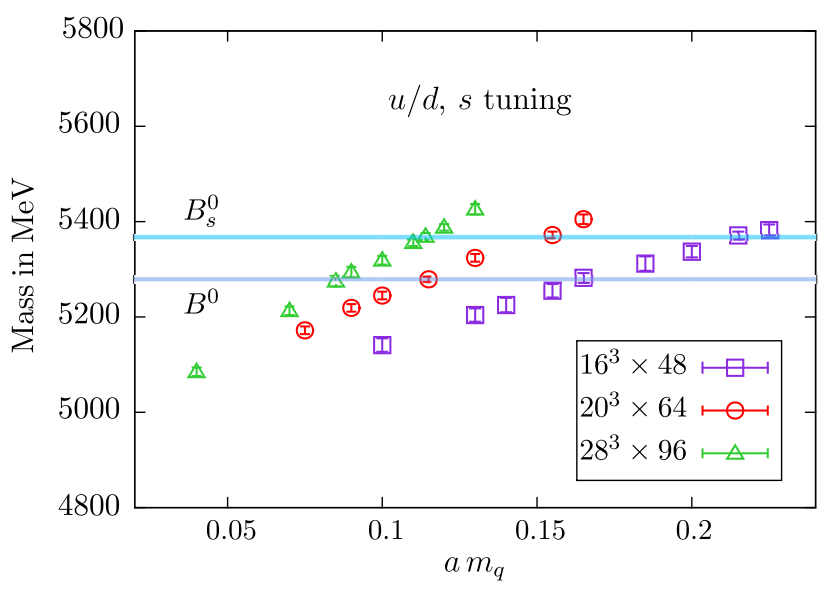

FIG. 1. Tuning of $s$ and $u / d$ quark masses in various lattices. The experimental values of $B^{0}$ and $B_{s}^{0}$ are shown by bands whose thickness are to enhance visibility and have nothing to do with experimental errors.

electromagnetic or isospin breaking in our calculation, we do not distinguish between $u$ and $d$ quarks, and it is always $a m_{u}=a m_{d}$. This tuning of $u / d$ mass to $B$ works well in capturing the $b$-baryon states containing a single $u / d$, such as $(u s b)$ or $(u c b)$ baryons, when compared to either PDG or other works.

In Table VI, we listed $u / d$ quark masses $\left(a m_{q}\right)$ against the lattice spacing. We show, in the Fig. 1, our strategy used to tune $m_{s}$ and $m_{u / d}$.

The tuned $a m_{u / d}$ for different lattices to use with $b$ quarks are

$$
16^{3} \times 48: \quad 0.165 \quad 20^{3} \times 64: 0.115 \quad 28^{3} \times 96: 0.085 .
$$

The $s$ and $u / d$ masses so tuned, $m_{s} / m_{u / d}$ turns out to be $\sim 1.3$ compared to $\sim 6$ that we get when $m_{s}$ is obtained from $\eta_{s}$ and $m_{u / d}$ is the bare sea quark mass.

For states containing two $u / d$ quarks, such as $\Sigma_{b}(u u b)$ and $\Lambda_{b}(u d b)$, we have mixed success with the above approach. When $b$ and $u$ form diquarks $\left(Q C \gamma_{\{k, 5\}} u\right)$ for $\Sigma_{b}$ state, the masses obtained are consistent with other lattice studies. However, this tuning scheme involving $B$ fails for $\Lambda_{b}$ where the diquark part is $\left(u C \gamma_{5} d\right)$ (see the Table II). Hence, for $\Lambda_{b}$ containing both $u$ and $d$ quarks, we have to resort to different tuning to account for $190 \mathrm{MeV}$ of mass difference with $\Sigma_{b}$. The mass of $\Lambda_{b}$ is obtained from this specially tuned $m_{u / d}^{\prime}$ (to be used only for $\Lambda_{b}$ ). Thus, tuned differently, the mass differences $\Xi_{b}-\Lambda_{b}$ and $\Sigma_{b}^{\star}-\Lambda_{b}$ are well within $2 \sigma$ of PDG values while $\Lambda_{b}-B$ is about $60 \mathrm{MeV}$ higher; see the Table XIV.

\section{RESULTS AND DISCUSSION}

In order to extract the masses of the bottom baryons, we perform a two-exponential uncorrelated fit to the two-point functions. We then cross-checked it with fitting the

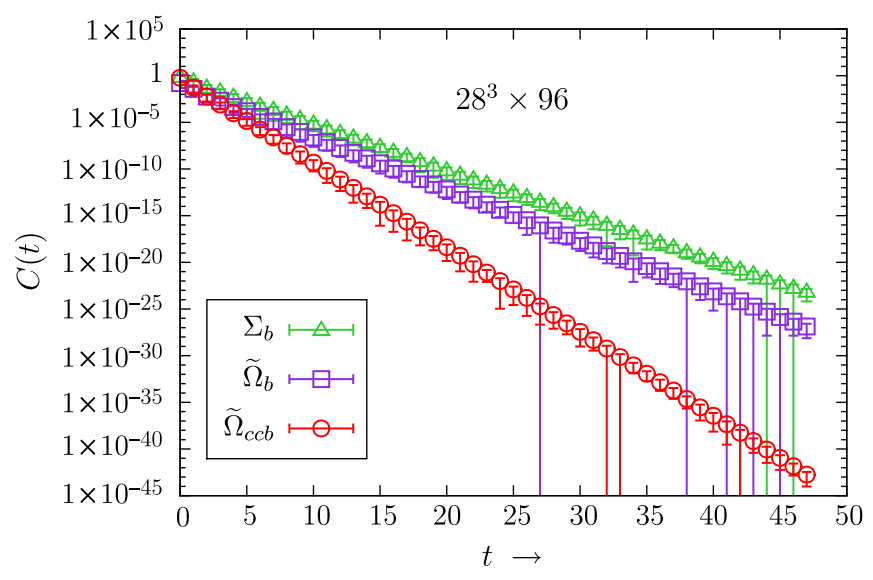

FIG. 2. $\Sigma_{b}, \tilde{\Omega}_{b}$ and $\tilde{\Omega}_{c c b}$ correlators in $28^{3} \times 96$ lattice.

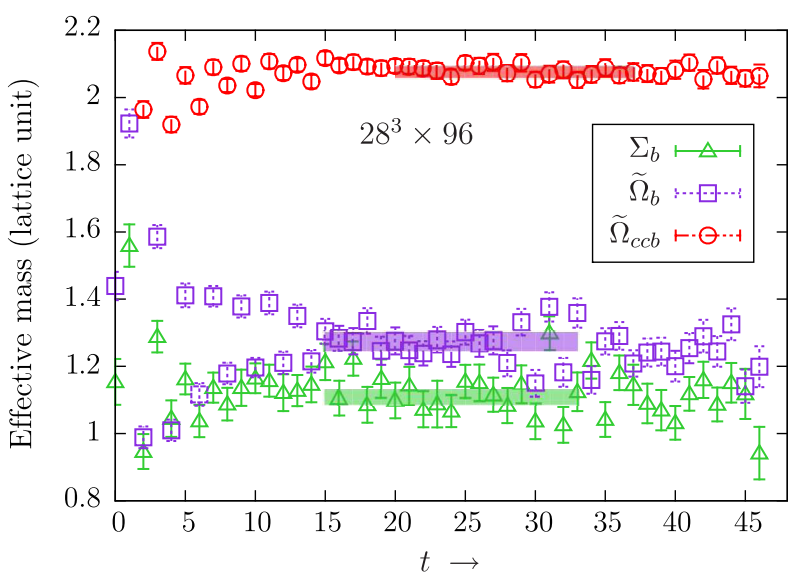

FIG. 3. Effective mass plots for the states in Fig. 2. The bands are placed over what we consider plateau.

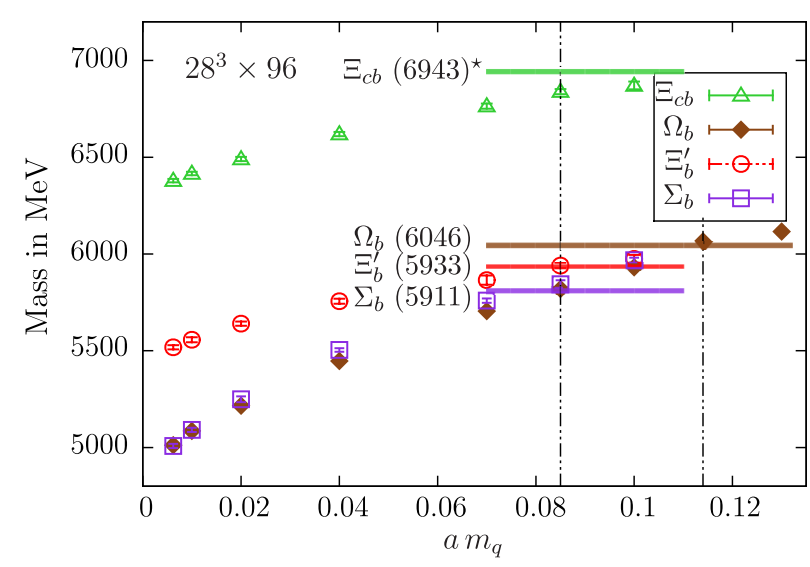

FIG. 4. Variation of single $b$ baryon masses in $\mathrm{MeV}$ against the same light quark masses as in Fig. $1 . m_{q}=0.085$ and 0.114 are the tuned $u / d$ and $s$ quark masses, respectively, indicated by dashed vertical lines. The bands correspond to the PDG values, except for $\Xi_{c b}$, which is taken from [11]. 
effective masses over the same range of time slices. However, this zero momentum energy does not directly give us the mass of the bottom baryons because of unphysical shift in zero of energy. To account for it, the mass is obtained considering energy splittings,

$$
M_{\text {latt }}=E_{\text {latt }}+\frac{n_{b}}{2}\left(M_{\text {phys }}^{\text {mod }}-E_{\text {latt }}^{\eta_{b}}\right),
$$

where $E_{\text {latt }}$ is the lattice zero momentum energy in $\mathrm{MeV}$ and $n_{b}$ is the number of $b$ quarks in the bottom baryon. For bottom mesons $(b \bar{l}), n_{b}$ is obviously always 1 . As discussed before, $M_{\text {phys }}^{\text {mod }}$ is the modified spin average mass of
$\Upsilon$ and $\eta_{b}$ and is equal to $9450 \mathrm{MeV}$, and $M_{\text {latt }}$ is the lattice bottom baryon mass in $\mathrm{MeV}$.

In the calculation of mass splittings, this shift in energies is canceled by subtraction among energies of hadrons having equal number of $b$ quarks $\left(n_{b}\right)$ in them. For this calculation, we use a jackknifed ratio of the correlation functions for fitting [34],

$$
C^{Y-X}(t)=\frac{C^{Y}(t)}{C^{X}(t)} \sim e^{-\left(M_{Y}-M_{X}\right) t}
$$

Below in the Fig. 2, we show a few correlators for single $b$ baryons containing exclusively either two $c$ or $s$ or $u / d$.

TABLE VII. Masses, in lattice unit, of baryons involving single $b$ quark and no $s$ quark. The bare $u / d$-quark masses are 0.165 for $16^{3} \times 48,0.115$ for $20^{3} \times 64$, and 0.085 for $28^{3} \times 96$.

\begin{tabular}{lcccc}
\hline \hline Baryons & $16^{3} \times 48(0.15 \mathrm{fm})$ & $20^{3} \times 64(0.12 \mathrm{fm})$ & $28^{3} \times 96(0.09 \mathrm{fm})$ & Average $(\mathrm{MeV})$ \\
\hline$\tilde{\Omega}_{c c b}^{\star}$ & $2.954(5)$ & $2.497(4)$ & $2.088(3)$ & $7807(11)$ \\
$\tilde{\Omega}_{c c b}$ & $2.933(5)$ & $2.482(3)$ & $2.078(3)$ & $7780(9)$ \\
$\Omega_{c c b}^{\prime}$ & $2.952(4)$ & $2.497(3)$ & $2.078(3)$ & $7797(11)$ \\
$\tilde{\Xi}_{c b}^{\star}$ & $1.899(4)$ & $1.648(6)$ & $6835(20)$ \\
$\Xi_{c b}$ & $2.222(6)$ & $1.881(4)$ & $1.623(5)$ & $6787(12)$ \\
$\tilde{\Xi}_{c b}$ & $2.177(11)$ & $1.886(4)$ & $1.631(4)$ & $6805(16)$ \\
$\Xi_{c b}^{\prime}$ & $1.904(4)$ & $1.653(6)$ & $6843(19)$ \\
$\tilde{\Sigma}_{b}^{\star}$ & $2.199(8)$ & $1.292(5)$ & $1.189(5)$ & $5836(22)$ \\
$\tilde{\Sigma}_{b}$ & $2.227(6)$ & $1.290(3)$ & $1.174(6)$ & $5820(21)$ \\
$\Sigma_{b}^{\prime}$ & $1.468(8)$ & $1.305(3)$ & $1.194(9)$ & $5848(18)$ \\
$\Lambda_{b}$ & $1.460(7)$ & $1.208(6)$ & $1.109(9)$ & $5667(14)$ \\
\hline \hline
\end{tabular}

TABLE VIII. Masses, in lattice unit, of baryons containing single $b$ quark and $s$ quark(s).

\begin{tabular}{lccccc}
\hline \hline Baryons & Tuning & $16^{3} \times 48(0.15 \mathrm{fm})$ & $20^{3} \times 64(0.12 \mathrm{fm})$ & $28^{3} \times 96(0.09 \mathrm{fm})$ & Average $(\mathrm{MeV})$ \\
\hline$\tilde{\Omega}_{c b}^{\star}$ & $\eta_{s}$ & $2.035(5)$ & $1.782(5)$ & $1.542(3)$ & $6611(9)$ \\
$\Omega_{c b}$ & $B_{s}$ & $2.292(7)$ & $1.957(6)$ & $1.693(4)$ & $6930(19)$ \\
& $\eta_{s}$ & $2.010(8)$ & $1.754(5)$ & $1.532(3)$ & $6578(9)$ \\
$\tilde{\Omega}_{c b}$ & $B_{s}$ & $2.248(11)$ & $1.937(7)$ & $1.684(2)$ & $6893(16)$ \\
& $\eta_{s}$ & $2.012(7)$ & $1.765(5)$ & $1.536(3)$ & $6587(10)$ \\
$\Omega_{c b}^{\prime}$ & $B_{s}$ & $2.267(8)$ & $1.943(7)$ & $1.686(2)$ & $6906(17)$ \\
& $\eta_{s}$ & $2.052(5)$ & $1.785(5)$ & $1.548(3)$ & $6625(8)$ \\
$\tilde{\Xi}_{b}^{\star}$ & $B_{s}$ & $2.297(6)$ & $1.966(6)$ & $1.705(2)$ & $6946(17)$ \\
& $\eta_{s}$ & $0.987(4)$ & $0.945(2)$ & $0.918(3)$ & $5237(8)$ \\
$\Xi_{b}$ & $B_{s}$ & $1.541(8)$ & $1.352(6)$ & $1.235(6)$ & $5935(22)$ \\
& $\eta_{s}$ & $0.986(5)$ & $0.947(2)$ & $0.909(4)$ & $5231(11)$ \\
$\tilde{\Xi}_{b}$ & $B_{s}$ & $1.520(9)$ & $1.345(3)$ & $1.207(6)$ & $5901(20)$ \\
& $\eta_{s}$ & $0.978(5)$ & $0.944(2)$ & $0.904(5)$ & $5222(13)$ \\
$\Xi_{b}^{\prime}$ & $B_{s}$ & $1.532(11)$ & $1.350(4)$ & $1.224(4)$ & $5921(19)$ \\
& $\eta_{s}$ & $0.987(4)$ & $0.948(3)$ & $0.913(5)$ & $5235(11)$ \\
$\tilde{\Omega}_{b}^{\star}$ & $B_{s}$ & $1.544(10)$ & $1.366(4)$ & $1.238(6)$ & $5946(16)$ \\
$\tilde{\Omega}_{b}$ & $\eta_{s}$ & $1.129(5)$ & $1.058(3)$ & $1.012(4)$ & $5430(11)$ \\
& $B_{s}$ & $1.611(8)$ & $1.412(6)$ & $1.264(3)$ & $6019(20)$ \\
$\Omega_{b}^{\prime}$ & $\eta_{s}$ & $1.118(7)$ & $1.050(3)$ & $0.997(4)$ & $5410(10)$ \\
& $B_{s}$ & $1.600(11)$ & $1.411(7)$ & $1.264(3)$ & $6014(17)$ \\
\hline & $\eta_{s}$ & $1.131(9)$ & $1.057(3)$ & $1.007(2)$ & $5427(9)$ \\
& $B_{s}$ & $1.615(8)$ & $1.425(7)$ & $1.295(2)$ & $6051(15)$ \\
\hline
\end{tabular}


The fitting range is typically chosen (i) looking at positions of what we consider plateau in the effective mass plots and (ii) exponential fits of the correlators. Both fittings return same masses over suitably chosen range. In the effective mass plot Fig. 3, the zero momentum energies and the errors of the same three states as in the Fig. 2 are represented as bands.

In these figures, we choose to present the data from $28^{3} \times 96$ lattices but the data from $16^{3} \times 48$ and $20^{3} \times 64$ are similar. Just to remind, in order to obtain the masses in $\mathrm{MeV}$ from these, we need the Eq. (41).

\section{A. Single bottom baryons}

A couple of baryon states containing one $b$ quark have been listed in the PDG [8], such as $\Lambda_{b}(u d b), \Omega_{b}(s s b)$, $\Xi_{b}^{\prime}(u s b)$ etc., and they provide a good matching opportunity. In the Fig. 4, we show the agreement of some of these baryon masses with PDG at the tuned $m_{u / d}$ and $m_{s}$.

In the following Tables VII, VIII, X, and XI, we present our results of the single and multi $b$ baryon states corresponding to the operators given in the Tables I and II. In the columns corresponding to various lattice ensembles, we show the masses in lattice unit, $a E_{\text {latt }}$ of the Eq. (41). In the last column of each table, we provide the average $M_{\text {latt }}$ and the statistical errors, calculated assuming the lattice configurations of different lattice spacings are statistically uncorrelated. Additionally, we include Tables XV and XVI for $M_{\text {latt }}$ for the bottom baryons for each lattice spacing in the Appendix.

We collect our results for single bottom baryons, not containing $s$ quark(s), in the Table VII and those with $s$ quark(s) in Table VIII. For the $m_{u / d}$, we state the results when the valence $m_{l}$ gives physical $B$ meson mass.

Since $s$ quark has been tuned in two different ways, we quote both the $b$-baryon masses at $\eta_{s}, B_{s}$ points.

As is evident from our results, the numbers coming from the $s$ quark tuned to $\eta_{s}$ are about $300 \mathrm{MeV}$ smaller from those tuned to $B_{s}(600 \mathrm{MeV}$ in baryons with two $s)$. If we take $\Omega_{b}(s s b)$ and compare with the PDG value $6046 \mathrm{MeV}$, it becomes obvious.

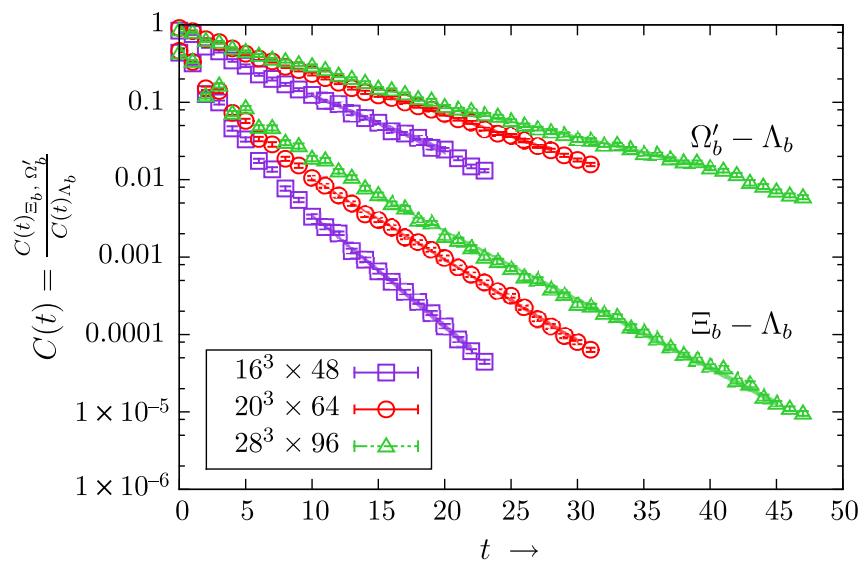

FIG. 5. The ratio of correlators for the calculation of the two splittings shown in Table IX. The bands overlaid on the data points represent single exponential fits.

Next, we determine mass differences in single bottom sector, including the hyperfine splittings.

The mass splittings are calculated using a ratio of correlators as given in the Eq. (42). As an example, in Fig. 5, we provide the plots for the ratio of correlators, $\Omega_{b}^{\prime}-\Lambda_{b}$ and $\Xi_{b}-\Lambda_{b}$, for comfortable viewing because of their relatively large mass differences; i.e., slopes are prominent and well separated. In the case of smaller differences, for instance, $\tilde{\Omega}_{b}^{\star}-\tilde{\Omega}_{b}$ or $\tilde{\Xi}_{c b}^{\star}-\tilde{\Xi}_{c b}$, the slopes of the ratio of correlators are rather small and not quite visible. In the Table IX above, we collect the results of single $b$ baryons mass splittings.

A heavy quark basically acts as a static color source, and therefore, we expect that the hyperfine splittings between states containing single or multiple $s$ and $u / d$ quark(s) to depend only weakly on the tuning of $m_{s}$ and $m_{u / d}$. For $m_{u / d} \leq 0.085$ and two values of $m_{s}$, we show this pattern for a few hyperfine splittings in Fig. 6.

\section{B. Double bottom baryons}

For the baryons containing more than one $b$ quark, the data are relatively less noisy than those containing a single

TABLE IX. Single bottom baryons mass splittings in MeV.

\begin{tabular}{lcccc}
\hline \hline Baryon splittings & $16^{3} \times 48(\mathrm{MeV})$ & $20^{3} \times 64(\mathrm{MeV})$ & $28^{3} \times 96(\mathrm{MeV})$ & Average (MeV) \\
\hline$\tilde{\Omega}_{c c b}^{\star}-\tilde{\Omega}_{c c b}$ & $28(3)$ & $23(2)$ & $\ldots$ & $26(3)$ \\
$\tilde{\Omega}_{c b}^{\star}-\tilde{\Omega}_{c b}$ & $59(8)$ & $62(13)$ & $61(22)$ & $61(15)$ \\
$\tilde{\Xi}_{c b}^{\star}-\tilde{\Xi}_{c b}$ & $37(6)$ & $44(5)$ & $44(9)$ & $42(7)$ \\
$\tilde{\Omega}_{b}^{\star}-\tilde{\Omega}_{b}$ & $29(5)$ & $28(11)$ & $29(4)$ & $29(7)$ \\
$\Omega_{b}^{\prime}-\Lambda_{b}$ & $396(4)$ & $391(9)$ & $406(10)$ & $398(9)$ \\
$\tilde{\Xi}_{b}^{\star}-\Xi_{b}$ & $138(20)$ & $122(38)$ & $138(46)$ & $133(36)$ \\
$\tilde{\Xi}_{b}-\Lambda_{b}$ & $170(9)$ & $166(11)$ & $163(6)$ & $166(9)$ \\
$\Lambda_{b}-B$ & $391(20)$ & $431(20)$ & $397(22)$ & $406(21)$ \\
$\tilde{\Sigma}_{b}^{\star}-\tilde{\Sigma}_{b}$ & $30(9)$ & $30(8)$ & $29(8)$ & $30(8)$ \\
$\tilde{\Sigma}_{b}^{\star}-\Lambda_{b}$ & $224(13)$ & $203(12)$ & $175(13)$ & $201(13)$ \\
\hline \hline
\end{tabular}




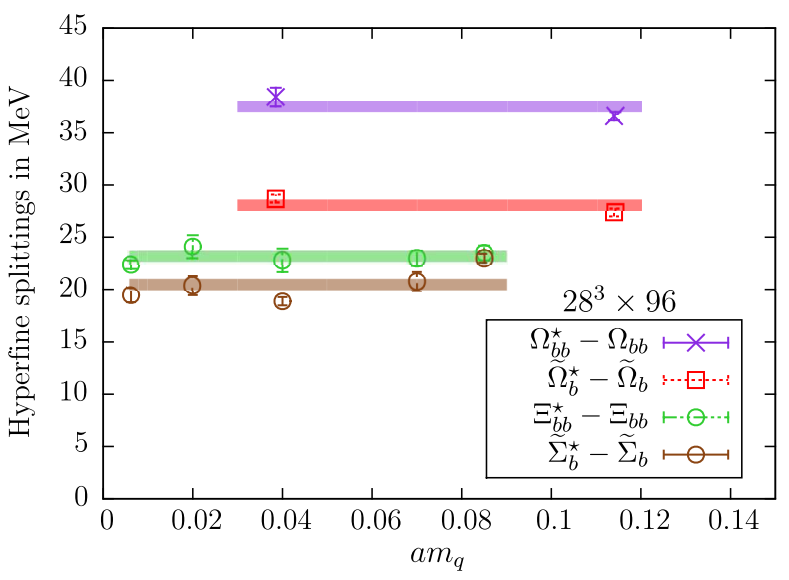

FIG. 6. Hyperfine splittings at various $m_{s}$ and $m_{u / d}$ for a selected few bottom baryons on $28^{3} \times 96$ lattice. Horizontal bands are the average values of the splittings and are used to guide the eye.

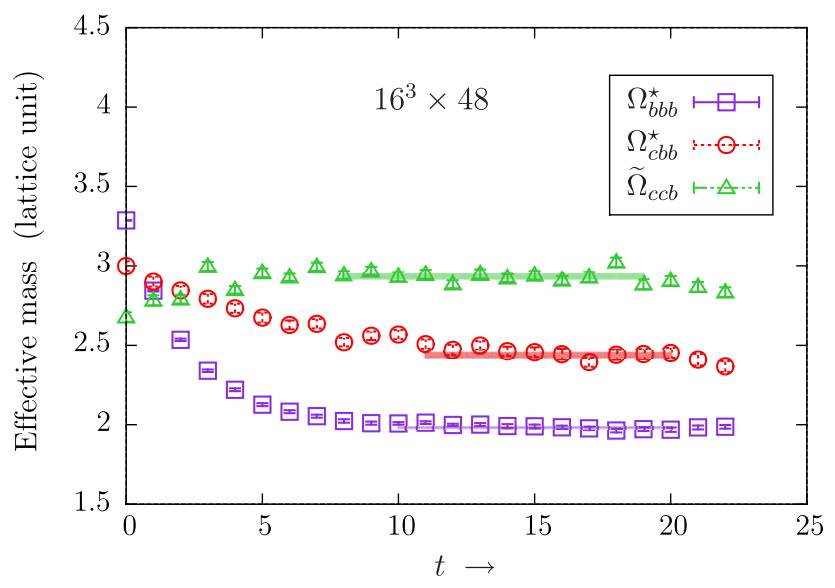

FIG. 7. $\Omega_{b b b}^{\star}, \tilde{\Omega}_{c b b}^{\star}$ and $\tilde{\Omega}_{c c b}$ effective masses.

$b$. The effective mass plots in Fig. 7, shown for only $16^{3} \times$ 48 lattices but similar for two other lattices, are an evidence for this. The fitting ranges are chosen the same way as is done for Fig. 3.
The plot for $\Omega_{b b b}^{\star}$ appears counterintuitive since being the heaviest, it is showing a lower mass compared to the other two. However, it receives a large correction because of shift in the rest mass of three $b$ quarks.

We tabulate our results for double bottom nonstrange baryons in the Table X, while those containing a $s$ quark in Table XI.

It is to note that $\Omega_{b b b}$ is a spin-3/2 state having no spin$1 / 2$ counterpart. But in practice, we can take a spin-1/2 projection to get such a fictitious state. Therefore, we label the physical $(b b b)$ spin-3/2 state with $\Omega_{b b b}^{\star}$ to keep consistency with our remaining notation. In this case, none of the states have PDG entries.

We would like to point out that the variation of the $\Xi_{b b}(u b b)$ masses with $m_{u / d}$ is almost absent as the major contribution to these baryons are coming from the two $b$ quarks. Similarly, from the Table XI, we see that the different tuning of the $s$ quark has significantly less influence on the double bottom baryon masses, a situation unsurprisingly similar to double bottom baryons with a $u / d$ quark.

The splittings in double bottom sector are tabulated in the Table XII.

In the double bottom sector, the splittings between the spin-3/2 and $1 / 2$ states are particularly interesting because HQET relates this mass differences with hyperfine splittings of heavy-light mesons, which in the heavy-quark limit [35],

$$
\frac{\Delta M_{b b}^{\text {baryon }}}{\Delta m_{b}^{\text {meson }}} \rightarrow \frac{3}{4}
$$

This behavior is consistent with our results within errors as can be seen in Table XIII.

A few GMO mass relations involving $b$ quarks are provided in Ref. [36], which we try to verify in this work,

$$
M_{\Omega_{c c b}^{\star}}-M_{\Omega_{c c b}} \approx M_{\Omega_{c b b}^{\star}}-M_{\Omega_{c b b}}
$$

TABLE X. Triple and double bottom nonstrange baryon masses.

\begin{tabular}{lcccc}
\hline \hline Baryon & $16^{3} \times 48(0.15 \mathrm{fm})$ & $20^{3} \times 64(0.12 \mathrm{fm})$ & $28^{3} \times 96(0.09 \mathrm{fm})$ & Average $(\mathrm{MeV})$ \\
\hline$\Omega_{b b b}^{\star}$ & $1.983(4)$ & $2.031(3)$ & $2.154(4)$ & $14403(7)$ \\
$\Omega_{b b b}$ & $1.974(4)$ & $2.023(5)$ & $2.148(4)$ & $14390(8)$ \\
$\Omega_{c b b}^{\star}$ & $2.429(16)$ & $2.259(4)$ & $2.117(3)$ & $11081(21)$ \\
$\Omega_{c b b}$ & $2.409(16)$ & $2.246(5)$ & $2.110(3)$ & $11060(23)$ \\
$\tilde{\Omega}_{c b b}^{\star}$ & $2.431(8)$ & $2.255(4)$ & $2.113(3)$ & $11077(14)$ \\
$\tilde{\Omega}_{c b b}^{\prime}$ & $2.432(10)$ & $2.251(4)$ & $2.113(3)$ & $11075(13)$ \\
$\Omega_{c b b}^{\prime}$ & $2.434(8)$ & $2.250(4)$ & $2.114(4)$ & $11076(12)$ \\
$\Xi_{b b}^{\star}$ & $1.721(12)$ & $1.643(10)$ & $1.666(5)$ & $10103(24)$ \\
$\Xi_{b b}$ & $1.700(12)$ & $1.640(7)$ & $1.664(5)$ & $10091(17)$ \\
$\tilde{\Xi}_{b b}^{\star}$ & $1.720(12)$ & $1.635(8)$ & $1.668(3)$ & $10100(27)$ \\
$\tilde{\Xi}_{b b}$ & $1.703(16)$ & $1.634(8)$ & $1.661(4)$ & $10087(22)$ \\
$\Xi_{b b}^{\prime}$ & $1.704(16)$ & $1.635(10)$ & $1.672(3)$ & $10096(24)$ \\
\hline \hline
\end{tabular}


TABLE XI. Double bottom strange baryon spectra.

\begin{tabular}{lccccc}
\hline \hline Baryon & Tuning & $16^{3} \times 48(0.15 \mathrm{fm})$ & $20^{3} \times 64(0.12 \mathrm{fm})$ & $28^{3} \times 96(0.09 \mathrm{fm})$ & Average $(\mathrm{MeV})$ \\
\hline$\Omega_{b b}^{\star}$ & $\eta_{s}$ & $1.545(11)$ & $1.536(6)$ & $1.576(4)$ & $9902(12)$ \\
& $B_{s}$ & $1.791(12)$ & $1.703(11)$ & $1.716(3)$ & $10203(22)$ \\
$\Omega_{b b}$ & $\eta_{s}$ & $1.553(9)$ & $1.527(7)$ & $1.570(4)$ & $9896(13)$ \\
& $B_{s}$ & $1.768(12)$ & $1.699(8)$ & $1.715(3)$ & $10190(17)$ \\
$\tilde{\Omega}_{b b}^{\star}$ & $\eta_{s}$ & $1.542(9)$ & $1.529(7)$ & $1.575(4)$ & $9896(12)$ \\
& $B_{s}$ & $1.791(12)$ & $1.693(10)$ & $1.717(3)$ & $10199(28)$ \\
$\tilde{\Omega}_{b b}$ & $\eta_{s}$ & $1.541(12)$ & $1.527(7)$ & $1.571(4)$ & $9891(13)$ \\
& $B_{s}$ & $1.789(9)$ & $1.695(7)$ & $1.714(3)$ & $10197(24)$ \\
$\Omega_{b b}^{\prime}$ & $\eta_{s}$ & $1.552(9)$ & $1.539(7)$ & $1.578(3)$ & $9908(11)$ \\
\hline \hline
\end{tabular}

TABLE XII. Double bottom baryon mass splittings in MeV. None of the splittings have PDG entries.

\begin{tabular}{lcccc}
\hline \hline Baryon splittings & $16^{3} \times 48(\mathrm{MeV})$ & $20^{3} \times 64(\mathrm{MeV})$ & $28^{3} \times 96(\mathrm{MeV})$ & Average $(\mathrm{MeV})$ \\
\hline$\tilde{\Omega}_{c b b}^{\star}-\tilde{\Omega}_{c b b}$ & $\ldots$ & $25(5)$ & $35(2)$ & $30(5)$ \\
$\Omega_{b b}^{\star}-\Omega_{b b}$ & $34(5)$ & $25(8)$ & $37(9)$ & $32(7)$ \\
$\Xi_{b b}^{\star}-\Xi_{b b}$ & $\cdots$ & $25(4)$ & $39(7)$ & $32(5)$ \\
\hline \hline
\end{tabular}

TABLE XIII. Ratio of hyperfine splittings of doubly heavy baryons to heavy mesons in the heavy quark limit in $28^{3} \times 96$ lattice.

\begin{tabular}{lcccc}
\hline \hline Baryon splittings & Our results $(\mathrm{MeV})$ & Meson splittings & Our results (MeV) & Ratio \\
\hline$\tilde{\Omega}_{b b c}^{\star}-\tilde{\Omega}_{b b c}$ & $35(2)$ & $B_{c}^{\star}-B_{c}$ & $46(4)$ & $0.76(4)$ \\
$\Omega_{b b}^{\star}-\Omega_{b b}$ & $37(9)$ & $B_{s}^{\star}-B_{s}$ & $45(9)$ & $0.82(9)$ \\
$\Xi_{b b}^{\star}-\Xi_{b b}$ & $39(7)$ & $B^{\star}-B$ & $47(7)$ & $0.83(8)$ \\
\hline \hline
\end{tabular}

$$
M_{\Sigma_{b}^{\star}}-M_{\Sigma_{b}} \approx M_{\Xi_{b b}^{\star}}-M_{\Xi_{b b}}
$$

For the GMO relation (44), both sides are expected to be approximately $31 \mathrm{MeV}$. In our case, for $20^{3} \times 64$ lattice, for which we have data for both the sides, they are approximately equal but are around $24 \mathrm{MeV}$ as against $31 \mathrm{MeV}$ given in [36]. Our lattice data are also consistent with the approximate GMO relation (45), where each side is about $30 \mathrm{MeV}$ against $20 \mathrm{MeV}$ calculated in [36].

\section{SUMMARY}

In this paper, we presented the lattice QCD determination of masses of the baryons containing one or more $b$ quark(s) using NRQCD action for the $b$ quark and HISQ action for the $c, s$, and $u / d$ quarks. This combination of NRQCD and HISQ has previously been employed in [16] for bottom mesons; however, the exact implementation was rather different. In this work, we converted the one component HISQ propagators to $4 \times 4$ matrices by the Kawamoto-Smit transformation and the two component NRQCD propagators to $4 \times 4$ matrices using the prescription suggested in [11].
We have discussed the construction of one and two bottom baryon operators in details and pointed out the difficulty for constructing operators motivated by HQET for single bottom baryons. Consequently, we modified the operators accordingly. For some of the baryons, we have multiple operators for the same state, i.e., baryons having the same quantum numbers. It would be natural in such cases to construct correlation matrices and obtain the lowest lying, i.e., ground states by solving the generalized eigenvalue method.

Single bottom baryons can have isodoublets with the same overall quantum numbers $J^{P}$. For instance, there exist three isodoublets of $\Xi_{b}$, which are not radially or orbitally excited states [37]. These states have been categorized by the spin of the $u s$ or $d s$ diquark denoted by $j$ and the spinparity of the baryon. These baryons are referred to as $\Xi_{b}\left(j=0, J^{P}=\frac{1}{2}^{+}\right), \Xi_{b}^{\prime}\left(j=1, J^{P}=\frac{1}{2}^{+}\right)$, and $\Xi_{b}^{\star}(j=1$, $\left.J^{P}=\frac{3}{2}^{+}\right)$. The same pattern is observed in $\Xi_{c}$ states [8]. The mass difference between $\Xi_{b}^{\prime}$ and $\Xi_{b}$ is about $150 \mathrm{Mev}$. So depending upon the choice of the wave function having the same overall quantum numbers, we can have different baryon states. If we choose $\left(s^{T} C \gamma_{5} d\right) Q$ as our $j=0$ baryon operator, then we will be simulating $\Xi_{b}$ state, and if we 


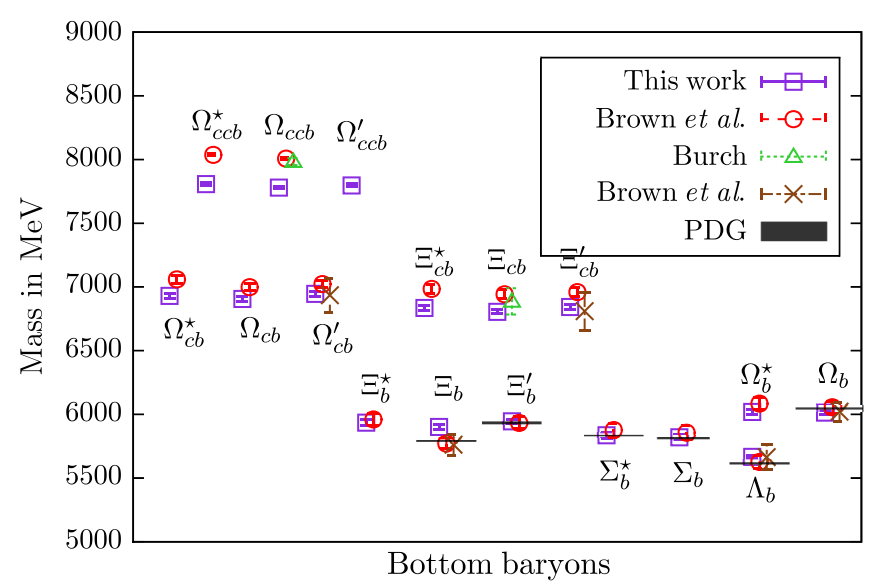

FIG. 8. Comparison of our single bottom baryon spectra with Brown et al. [11], Burch [10], Mathur et al. [6], and PDG [8], where available.

project out the spin- $1 / 2$ state of $j=1$ operator $\left(s^{T} C \gamma_{k} d\right) Q$, then we will get the $\Xi_{b}^{\prime}$. For the reason discussed before, we can not define $j=1$ light-light diquark state. In our case, the wave function corresponds to $\Xi_{b}^{\prime}$ is $\left(Q^{T} C \gamma_{5} s\right) d$. Constructing an operator in this way allows the $s$ and $d$ quarks to have parallel spin configurations. By simple physical reasoning, we can argue that the explicit construction of $j=0$ diquark for $\Xi_{b}$ is more likely to have a significant overlap with physical $\Xi_{b}$ compared to $\Xi_{b}^{\prime}(j=1)$ upon gauge averaging. However, the operator $\left(Q^{T} C \gamma_{5} s\right) d$ is expected to have a good overlap with the $\Xi_{b}^{\prime}$ state, and this is also supported by our result. For an antiparallel $s$ and $d$ spin configuration, $\Xi_{b}^{\prime}$ can also have an overlap with the $\Xi_{b}$ state. On lattice, operators for states having the same quantum numbers can mix, and therefore, a detailed generalized eigenvalue problem analysis can only resolve the issue of mutual overlap of $\Xi_{b}$ and $\Xi_{b}^{\prime}$ states, which we did not include in this work. This is perhaps the reason we see discrepancies in their values with PDG and others in Fig. 8.

The $b$ mass has been tuned to modified $\Upsilon-\eta_{b}$ spin average mass, while $c$ quark to $J / \psi-\eta_{c}$ spin average mass. The $s$ quark is required to be tuned to both the fictitious $\eta_{s}$ and $B_{s}$ mass since we expect the bottomstrange bound state to be more appropriate than the $s-\bar{s}$ bound state in bottom baryons. For the light $u / d$ quarks, we have considered a wide range of bare masses and tune it using $B$ meson. However, this scheme of tuning $u / d$ quarks has not worked for $\Lambda_{b}$. There, $u / d$ are tuned to capture the $190 \mathrm{MeV}$ mass difference $\Sigma_{b}-\Lambda_{b}$. This specially tuned $m_{u / d}^{\prime}$, which is used only for $\Lambda_{b}$, gives it a mass of $5667 \mathrm{MeV}$. The PDG value for $\Lambda_{b}$ mass is $5620 \mathrm{MeV}$. We demonstrated the variation of bottom baryons as well as hyperfine splittings against varying $m_{s}$ and $m_{u / d}$. We showed that the hyperfine splittings are almost independent of $s$ and $u / d$ quark masses.

We compare our bottom baryon results with other works, mostly with $[6,10,11]$, in Figs. 8 and 9. NRQCD has been

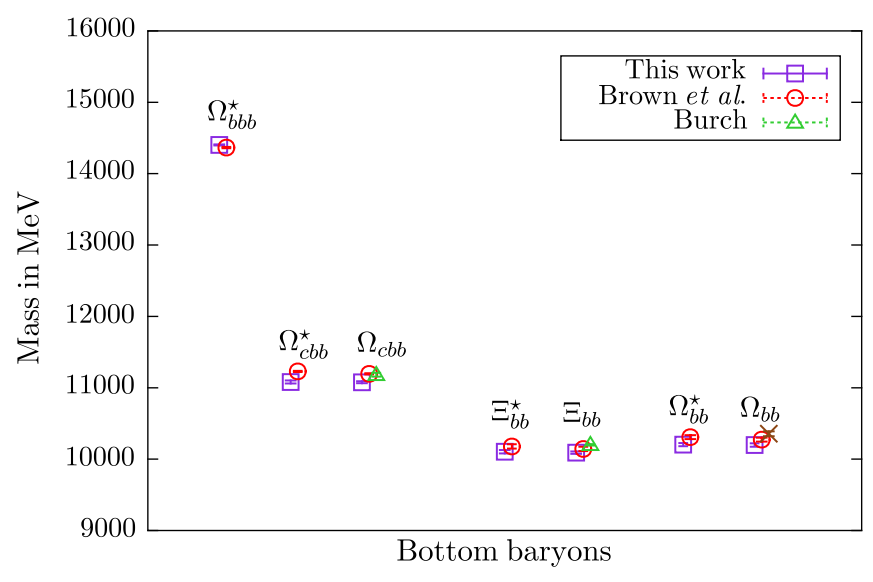

FIG. 9. Comparison of our triple and double bottom baryon spectra with Brown et al. [11] and Burch [10].

the standard action of choice for the $b$ quark in these three cited studies, but the actions used for $c$ are all differentNRQCD, Clover-Wilson, and relativistic heavy quark action [38]. Whatever differences we see in the results for single $b$ baryons with $c$ quark(s), particularly in the cases with two $c$, possibly have stemmed from the differences in actions. However, in this work, we do not address the systematics involved, which could be significant, because of these differences. (The study of such systematics needed to arrive at phenomenologically relevant numbers will be reported elsewhere.) But otherwise, the results of bottom baryon spectra in the present study with NRQCD $b$ quark and HISQ $c, s, u / d$ quarks appear to agree with each other. We would like to emphasize again that the errors we reported here are only statistical.

The comparison of the hyperfine splittings is shown in Fig. 10.

Apart from the hyperfine splittings, a few other mass splittings calculated in this work are assembled in Table XIV. The bottom baryon spectra and various mass splittings reported in this paper and those appearing in

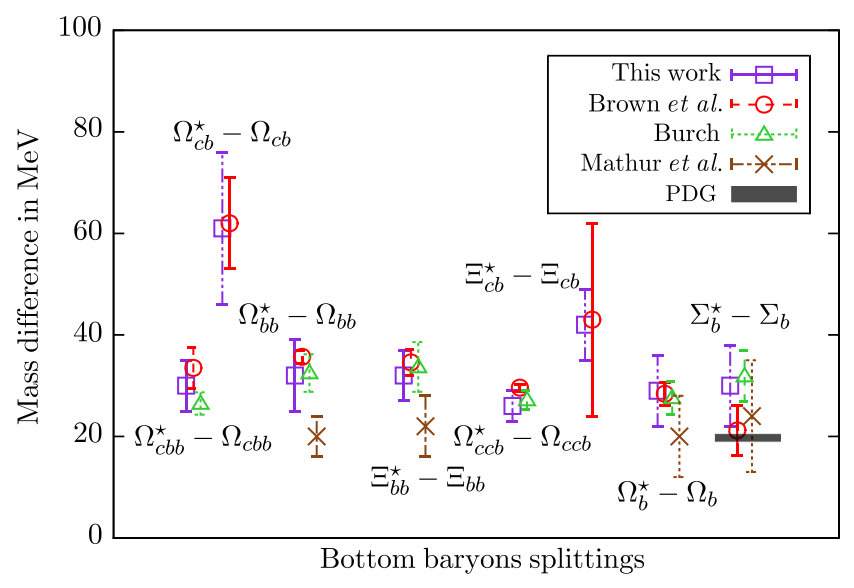

FIG. 10. Hyperfine splittings of bottom baryons calculated in this work and compared with Brown et al. [11], Burch [10], Mathur et al. [6], and PDG [8], where available. 
TABLE XIV. Bottom baryon mass differences in MeV. PDG values without error are simply the differences of the two states.

\begin{tabular}{lccc}
\hline \hline Mass splittings & This work & Brown et al. [11] & PDG [8] \\
\hline$\Omega_{b}^{\prime}-\Lambda_{b}$ & $398(9)$ & $\ldots$ & $426.4(2.2)$ \\
$\Xi_{b}^{\star}-\Xi_{b}$ & $133(36)$ & $189(29)$ & 155.5 \\
$\Xi_{b}-\Lambda_{b}$ & $166(9)$ & $\ldots$ & $172.5(0.4)$ \\
$\Lambda_{b}-B$ & $406(21)$ & $\ldots$ & $339.2(1.4)$ \\
$\Sigma_{b}^{\star}-\Lambda_{b}$ & $201(13)$ & $251(46)$ & 213.5 \\
\hline \hline
\end{tabular}

$[8,11]$ are well comparable given the wide choice of actions and tuning employed in achieving them.

\section{ACKNOWLEDGEMENTS}

The numerical part of this work has been performed at the HPC facility in NISER (Kalinga cluster) funded by
Department of Atomic Energy (DAE), Government of India. A significant part of this work has been carried out in the Proton cluster funded by DST-SERB Project No. SR/S2/HEP-0025/2010. The authors acknowledge useful discussions with Dipankar Chakrabarti (IIT-Kanpur, India) and Stefan Meinel (University of Arizona, U.S.) on bottom baryon operator construction. One of the authors (P. M.) thanks DAE for financial support.

\section{APPENDIX: BOTTOM BARYON MASSES IN MEV}

For the reason of completeness, we provide the bottom baryon masses in $\mathrm{MeV}$ for different lattices used in this work. In these tables, we provide only $B_{s}$ tuned strange bottom baryon masses and not $\eta_{s}$ tuned values.

TABLE XV. Single bottom baryon masses in MeV, corresponding to the Tables VII and VIII.

\begin{tabular}{|c|c|c|c|c|c|c|c|c|c|}
\hline Baryons & $\begin{array}{l}16^{3} \times 48 \\
(0.15 \mathrm{fm})\end{array}$ & $\begin{array}{l}20^{3} \times 64 \\
(0.12 \mathrm{fm})\end{array}$ & $\begin{array}{l}28^{3} \times 96 \\
(0.09 \mathrm{fm})\end{array}$ & Average & Baryons & $\begin{array}{l}16^{3} \times 48 \\
(0.15 \mathrm{fm})\end{array}$ & $\begin{array}{l}20^{3} \times 64 \\
(0.12 \mathrm{fm})\end{array}$ & $\begin{array}{l}28^{3} \times 96 \\
(0.09 \mathrm{fm})\end{array}$ & Average \\
\hline$\tilde{\Omega}_{c c b}^{\star}$ & $7816(6)$ & 7794(6) & $7805(6)$ & $7807(11)$ & $\tilde{\Omega}_{c b}^{\star}$ & $6945(9)$ & $6906(10)$ & 6938(9) & 6930(19) \\
\hline$\tilde{\Omega}_{c c b}$ & $7788(6)$ & $7769(5)$ & $7782(6)$ & $7780(9)$ & $\Omega_{c b}$ & $6887(14)$ & $6873(11)$ & 6919(4) & $6893(16)$ \\
\hline$\Omega_{c c b}^{\prime}$ & $7813(5)$ & 7794(5) & $7782(6)$ & $7797(11)$ & $\tilde{\Omega}_{c b}$ & $6912(10)$ & $6883(11)$ & 6923(4) & $6906(17)$ \\
\hline$\tilde{\Xi}_{c b}^{\star}$ & $6853(8)$ & $6810(6)$ & $6840(13)$ & $6835(20)$ & $\Omega_{c b}^{\prime}$ & $6951(8)$ & 6921(10) & $6965(4)$ & $6946(17)$ \\
\hline$\Xi_{c b}$ & 6794(14) & $6781(6)$ & $6785(11)$ & $6787(12)$ & $\tilde{\Xi}_{b}^{\star}$ & $5957(10)$ & $5911(10)$ & $5934(13)$ & $5935(22)$ \\
\hline$\tilde{\Xi}_{c b}$ & $6822(10)$ & $6789(6)$ & 6802(9) & $6805(16)$ & $\Xi_{b}$ & $5929(12)$ & $5900(5)$ & 5873(13) & $5901(20)$ \\
\hline$\Xi_{c b}^{\prime \prime}$ & $6859(8)$ & $6819(6)$ & 6851(13) & $6843(19)$ & $\tilde{\Xi}_{b}$ & $5945(14)$ & $5908(7)$ & $5910(9)$ & $5921(19)$ \\
\hline$\tilde{\Sigma}_{b}^{\star}$ & $5861(10)$ & $5812(8)$ & $5833(11)$ & $5836(22)$ & $\Xi_{b}^{\prime}$ & 5961(13) & $5934(7)$ & 5941(13) & $5946(16)$ \\
\hline$\tilde{\Sigma}_{b}$ & $5850(9)$ & $5809(5)$ & $5800(13)$ & $5820(21)$ & $\tilde{\Omega}_{b}^{\star}$ & $6049(10)$ & $6010(10)$ & $5998(7)$ & $6019(20)$ \\
\hline$\Sigma_{b}^{\prime}$ & $5864(9)$ & $5834(5)$ & $5844(20)$ & $5848(18)$ & $\tilde{\Omega}_{b}$ & $6035(14)$ & $6008(11)$ & $5998(7)$ & $6014(17)$ \\
\hline$\Lambda_{b}$ & $5669(9)$ & $5674(10)$ & $5658(20)$ & $5667(14)$ & $\Omega_{b}^{\prime}$ & $6054(10)$ & 6031(11) & $6066(4)$ & $6051(15)$ \\
\hline
\end{tabular}

TABLE XVI. Triple and double bottom baryon masses in $\mathrm{MeV}$, corresponding to the Tables X and XI.

\begin{tabular}{|c|c|c|c|c|c|c|c|c|c|}
\hline Baryons & $\begin{array}{c}16^{3} \times 48 \\
(0.15 \mathrm{fm})\end{array}$ & $\begin{array}{l}20^{3} \times 64 \\
(0.12 \mathrm{fm})\end{array}$ & $\begin{array}{c}28^{3} \times 96 \\
(0.09 \mathrm{fm})\end{array}$ & Average & Baryons & $\begin{array}{l}16^{3} \times 48 \\
(0.15 \mathrm{fm})\end{array}$ & $\begin{array}{l}20^{3} \times 64 \\
(0.12 \mathrm{fm})\end{array}$ & $\begin{array}{l}28^{3} \times 96 \\
(0.09 \mathrm{fm})\end{array}$ & Average \\
\hline$\Omega_{b b b}^{\star}$ & $14399(5)$ & $14405(5)$ & $14403(9)$ & $14403(7)$ & $\Omega_{b b}^{\star}$ & $10217(16)$ & $10177(18)$ & $10216(7)$ & $10203(22)$ \\
\hline$\Omega_{b b b}$ & $14388(5)$ & 14392(8) & $14390(9)$ & $14390(8)$ & $\Omega_{b b}$ & $10187(16)$ & 10171(13) & $10214(7)$ & $10190(17)$ \\
\hline$\Omega_{c b b}^{\star}$ & $11056(21)$ & 11091(6) & $11095(7)$ & $11081(21)$ & $\tilde{\Omega}_{b b}^{\star}$ & $10217(16)$ & 10161(16) & $10218(7)$ & $10199(28)$ \\
\hline$\Omega_{c b b}$ & $11029(21)$ & $11070(8)$ & $11080(7)$ & $11060(23)$ & $\tilde{\Omega}_{b b}$ & $10214(12)$ & $10164(11)$ & $10212(7)$ & $10197(24)$ \\
\hline$\tilde{\Omega}_{c h b}^{\star}$ & $11058(10)$ & $11085(7)$ & $11086(7)$ & $11077(14)$ & $\Omega_{b b}^{\prime}$ & $10205(12)$ & 10171(11) & $10225(7)$ & $10200(20)$ \\
\hline$\tilde{\Omega}_{c b b}$ & $11060(13)$ & 11078(7) & 11086(7) & $11075(13)$ & & & & & \\
\hline$\Omega_{c b b}^{\prime}$ & $11062(10)$ & $11076(7)$ & 11088(9) & $11076(12)$ & & & & & \\
\hline$\Xi_{b b}^{\star}$ & $10124(16)$ & $10078(16)$ & $10106(11)$ & $10103(24)$ & & & & & \\
\hline$\Xi_{b b}$ & $10097(16)$ & $10073(11)$ & $10102(11)$ & 10091(17) & & & & & \\
\hline$\tilde{\Xi}_{b b}^{\star}$ & $10123(16)$ & $10065(13)$ & 10111(7) & $10100(27)$ & & & & & \\
\hline$\tilde{\Xi}_{b b}$ & 10101(21) & $10063(13)$ & $10095(9)$ & $10087(22)$ & & & & & \\
\hline$\Xi_{b b}^{\prime}$ & $10102(21)$ & $10065(16)$ & $10119(7)$ & $10096(24)$ & & & & & \\
\hline
\end{tabular}


[1] C. T. H. Davies, Proc. Sci., LATTICE2011 (2011) 019.

[2] E. Gamiz, C. T. H. Davies, G. P. Lepage, J. Shigemitsu, and M. Wingate, Phys. Rev. D 80, 014503 (2009).

[3] A. A. Khan, T. Bhattacharya, S. Collins, C. T. H. Davies, R. Gupta, C. Morningstar, J. Shigemitsu, and J. Sloan, Phys. Rev. D 62, 054505 (2000).

[4] R. Woloshyn, Phys. Lett. B 476, 309 (2000).

[5] R. Lewis, N. Mathur, and R. M. Woloshyn, Phys. Rev. D 64, 094509 (2001).

[6] N. Mathur, R. Lewis, and R. M. Woloshyn, Phys. Rev. D 66, 014502 (2002).

[7] H. Na and S. A. Gottlieb, Proc. Sci., LATTICE2006 (2006) 191; LATTICE2007 (2007) 124; LATTICE2008 (2008) 119.

[8] M. Tanabashi et al. (Particle Data Group), Phys. Rev. D 98, 030001 (2018) and 2019 update.

[9] S. Meinel, Phys. Rev. D 82, 114514 (2010).

[10] T. Burch, arXiv:1502.00675.

[11] Z. S. Brown, W. Detmold, S. Meinel, and K. Orginos, Phys. Rev. D 90, 094507 (2014).

[12] G. P. Lepage, L. Magnea, C. Nakhleh, U. Magnea, and K. Hornbostel, Phys. Rev. D 46, 4052 (1992).

[13] B. A. Thacker and G. P. Lepage, Phys. Rev. D 43, 196 (1991).

[14] E. Follana, Q. Mason, C. Davies, K. Hornbostel, G. P. Lepage, J. Shigemitsu, H. Trottier, and K. Wong, Phys. Rev. D 75, 054502 (2007).

[15] A. X. El-Khadra, A. S. Kronfeld, and P. B. Mackenzie, Phys. Rev. D 55, 3933 (1997).

[16] E. B. Gregory, C. T. H. Davies, I. D. Kendall, J. Koponen, K. Wong, E. Follana, E. Gámiz, G. P. Lepage, E. H. Müller, H. $\mathrm{Na}$, and J. Shigemitsu (HPQCD Collaboration), Phys. Rev. D 83, 014506 (2011).

[17] www.physics.utah.edu/ detar/milc/.

[18] N. Kawamoto and J. Smit, Nucl. Phys. B192, 100 (1981).

[19] C. T. H. Davies, K. Hornbostel, A. Langnau, G. P. Lepage, A. Lidsey, J. Shigemitsu, and J. Sloan, Phys. Rev. D 50, 6963 (1994).

[20] C. T. H. Davies, K. Hornbostel, G. P. Lepage, A. J. Lidsey, J. Shigemitsu, and J. Sloan, Phys. Rev. D 52, 6519 (1995).
[21] H. D. Trottier, Phys. Rev. D 55, 6844 (1997).

[22] M. Wingate, J. Shigemitsu, C. T. H. Davies, G. P. Lepage, and H.D. Trottier, Phys. Rev. D 67, 054505 (2003).

[23] K. C. Bowler, R. D. Kenway, O. Oliveira, D. G. Richards, P. Ueberholz, L. Lellouch, J. Nieves, C. T. Sachrajda, N. Stella, and H. Wittig (UKQCD Collaboration), Phys. Rev. D 54, 3619 (1996).

[24] J. M. Flynn, F. Mescia, and A. S. B. Tariq, J. High Energy Phys. 07 (2003) 066.

[25] J. G. Korner, M. Kramer, and D. Pirjol, Prog. Part. Nucl. Phys. 33, 787 (1994).

[26] A. Bazavov et al., Rev. Mod. Phys. 82, 1349 (2010).

[27] K. Orginos and D. Toussaint, Phys. Rev. D 59, 014501 (1998).

[28] K. Orginos, D. Toussaint, and R. L. Sugar, Phys. Rev. D 60, 054503 (1999).

[29] N. Zerf, Proc. Sci., RADCOR2015 (2015) 089.

[30] T. C. Hammant, A. G. Hart, G. M. von Hippel, R. R. Horgan, and C. J. Monahan, Phys. Rev. Lett. 107, 112002 (2011).

[31] R. J. Dowdall, C. T. H. Davies, T. C. Hammant, and R. R. Horgan, Phys. Rev. D 86, 094510 (2012).

[32] C. Bernard, T. Burch, K. Orginos, D. Toussaint, T. A. DeGrand, C. DeTar, S. Datta, S. Gottlieb, U. M. Heller, and R. Sugar, Phys. Rev. D 64, 054506 (2001).

[33] C. T. H. Davies, E. Follana, I. D. Kendall, G. P. Lepage, and C. McNeile, Phys. Rev. D 81, 034506 (2010).

[34] S. R. Beane, K. Orginos, and M. J. Savage, Phys. Lett. B 654, 20 (2007).

[35] N. Brambilla, A. Vairo, and T. Rösch, Phys. Rev. D 72, 034021 (2005).

[36] X.-Z. Weng, X.-L. Chen, and W.-Z. Deng, Phys. Rev. D 97, 054008 (2018).

[37] R. Aaij et al. (LHCb Collaboration), Phys. Rev. Lett. 114, 062004 (2015).

[38] S. Aoki, Y. Kuramashi, and S. Tominaga, Prog. Theor. Phys. 109, 383 (2003). 